\title{
Photometric study of the young open cluster NGC $3293^{\star, \star \star}$
}

\author{
G. Baume ${ }^{1,2, \star \star \star}$, R. A. Vázquez ${ }^{1, \star \star \star}$, G. Carraro ${ }^{2}$, and A. Feinstein ${ }^{1}$ \\ 1 Facultad de Ciencias Astronómicas y Geofísicas de la UNLP, IALP-CONICET, Paseo del Bosque s/n, \\ 1900 La Plata, Argentina \\ 2 Dipartimento di Astronomia, Università di Padova, Vicolo Osservatorio 2, 35122 Padova, Italy
}

Received 27 September 2002 / Accepted 17 January 2003

\begin{abstract}
Deep and extensive CCD photometric observations at $U B V(R I)_{\mathrm{C}} \mathrm{H}_{\alpha}$ were carried out in the area of the open cluster NGC 3293. The new data set allows to see the entire cluster sequence down to $M_{V} \approx+4.5$, revealing that stars with $M_{V}<-2$ are evolving off the main sequence; stars with $-2<M_{V}<+2$ are located on the main sequence and stars with $M_{V}>+2$ are placed above it. According to our analysis, the cluster distance is $d=2750 \pm 250 \mathrm{pc}\left(V_{0}-M_{V}=12.2 \pm 0.2\right)$ and its nuclear age is $8 \pm 1$ Myr. NGC 3293 contains an important fraction of pre-main sequence (PMS) stars distributed along a parallel band to the ZAMS with masses from 1 to $2.5 \mathcal{M}_{\odot}$ and a mean contraction age of $10 \mathrm{Myr}$. This last value does not differ too much from the nuclear age estimate. If we take into account the many factors that may affect the PMS star positions on the colourmagnitude diagram, both ages can be perfectly reconciled. The star formation rate, on the other hand, suggests that NGC 3293 stars formed surely in one single event, therefore favouring a coeval process of star formation. Using the $\mathrm{H}_{\alpha}$ data, we detected nineteen stars with signs of $\mathrm{H}_{\alpha}$ emission in the region of NGC 3293, another indication that the star formation process is still active in the region. The computed initial mass function for the cluster has a slope of $x=1.2 \pm 0.2$, a bit flatter than the typical slope for field stars and similar to the values found for other young open clusters.
\end{abstract}

Key words. Galaxy: open clusters and associations: individual: NGC 3293 - stars: imaging - stars: luminosity function, mass function

\section{Introduction}

Star clusters constitute the most appropriate laboratory to test the stellar evolution theory since all the stars formed in a cluster belong to the same region of space, are all at the same distance and have the same chemical composition. Some of the main tools, tightly related to the history of the star formation processes, are the luminosity function (LF) and the initial mass function (IMF). Regarding the construction of these distributions, open clusters offer two operational advantages when compared to field stars: a) there is no need to assume a timeindependent IMF as necessary in deriving the IMF field stars and, b) as cluster stars do not move far from their birth-sites, neither is necessary to consider the IMF is spatially independent (Scalo 1986; Herbst \& Miller 1982; Wilner \& Lada 1991). Still, after several years of intensive and extensive works on

Send offprint requests to: $\mathrm{G}$. Baume, e-mail: gbaume@fcaglp.fcaglp.unlp.edu.ar

* Based on observations collected at UTSO, ESO (Dutch $0.9 \mathrm{~m}$ telescope) and CASLEO. The CCD and data acquisition system at CASLEO has been partly financed by R.M. Rich through U.S. NSF Grant AST-90-15827.

$\star \star$ Full Table 1 is only available in electronic form at the CDS via an anonymous ftp to cdsarc.u-strasbg.fr $(130.79 .128 .5)$ or via http://cdsweb.u-strasbg.fr/cgi-bin/qcat?]/A+A/402/549 $\star \star \star$ Visiting Astronomer, CASLEO operated under agreement between CONICET of the República Argentina and the National Universities of La Plata, Córdoba and San Juan. open clusters (in our galaxy and the Magellanic Clouds) applying the powerful $\mathrm{CCD}$ techniques, some questions remain unanswered:

- What the star formation mechanism and for how long is it active (Sung et al. 1998)? Is it coeval or sequential (Iben \& Talbot 1966; Herbst \& Miller 1982; Adams et al. 1983)?

- Is the IMF slope universal? Is it bimodal? Why do some open clusters show an apparent deficiency of low mass stars (van den Bergh \& Sher 1960; Adams et al. 1983; Lada et al. 1993; Phelps \& Janes 1993; Sung et al. 1998; Prisinzano et al. 2001), even though they are young enough to exclude stellar loss by dynamical evolution?

- Does the IMF vary from cluster to cluster even within the same star formation region, probably by changes in the initial conditions of the star formation process (Scalo 1986; Lada \& Lada 1995)?

The young open cluster NGC $3293=\mathrm{C} 1033-579\left(l=285.9^{\circ}\right.$, $\left.b=0.07^{\circ}\right)$ is placed in the Carina region north-west of Trumpler 14/16. The three clusters are embedded in the nebulosity of the HII region NGC 3372, although NGC 3293 is relatively free of patches of dust. This cluster is compact, well populated and not too reddened. With an age $<10 \mathrm{Myr}$, it is likely free from dynamical evolution too. Altogether these properties make it an excellent target to examine some of the items mentioned above. Herbst \& Miller (1982, hereafter HM82) performed the deepest photometric study (mostly photographic) of this object to investigate the star-forming 
history down to $V \approx 15$. HM82 found features that deserve confirmation: a) the low mass stars formed first, b) the cluster LF shows a sharp dip at $M_{V}=+2$ and +3 , c) the cluster IMF is not only flatter than the field star IMF but it varies with time during the cluster formation period and d) the cluster has a halo structure formed by less massive stars. The need for new deep photometry is evident as a substantial part of HM82's findings may have been produced by selection effects close to the detection limit of the photographic plates (Deeg \& Ninkov 1996).

Apart from the HM82 study of the star-forming history, a synthesis of the main investigations carried out in NGC 3293 includes: spectroscopy of bright cluster members to obtain radial velocities and spectral classification by Feast (1958); rotational velocity studies undertaken by Balona (1975); UBVRI photoelectric photometry and polarimetric measures performed by Feinstein \& Marraco (1980, hereafter FM80); $U B V$ photometry including the cluster and the surrounding area carried out by Turner et al. (1980, hereafter TGHH80); important contributions including the detection of several $\beta$ Cepheid stars come from $u b v y \mathrm{H}_{\beta}$ observations (Shobbrook 1980; Balona \& Engelbrecht 1981, 1983; Shobbrook 1983; Balona 1994).

The present investigation aims at defining the lower main sequence structure of this cluster and detecting the presence of pre-main sequence (PMS) stars. We understand that a primary (but not conclusive) indicator of the existence of PMS objects comes from the detection of faint cluster members above the ZAMS (Walker 1957, 1961). More recent evidence on PMS stars (cf. Fig. 3 in Preibisch \& Zinnecker 1999) indicates that they form a band $1^{\mathrm{m}}$ above the ZAMS, 1 to $2^{\mathrm{m}}$ wide approximately in the HR diagram. However, the determination of this locus through photometry alone is completely spoiled because of the contamination by field stars. Better defined locations are achieved by removing the field star contamination through adequate comparison fields. That implies that we need to obtain a "clean" colour-magnitude diagram (CMD), where the PMS stars, if they do exist, occupy a well-defined place. Another purpose of this investigation deals with the analysis of the frequency distribution of both magnitudes and masses, including not only the brightest (most massive) members but also the faintest ones. Finally we will attempt to detect $\mathrm{H}_{\alpha}$ emission stars (indicative of the PMS stage) through an $\mathrm{H}_{\alpha}$ (on/off) survey that was conducted in the NGC 3293 region.

In Sect. 2 we describe our observations, the reduction procedure and the complementary sources of information. Section 3 contains the data analysis including the estimation of the cluster angular size and the membership assignment. Section 4 illustrates the determination of the basic cluster parameters: reddening, distance, linear size and age; it also contains the cluster LF and IMF determination. Section 5 describes the process to detect stars with $\mathrm{H}_{\alpha}$ emission. In Sect. 6 we discuss the star formation process. Finally, the conclusions are included in Sect. 7 along with a summary of our main findings.

\section{Data set}

The main data set comes from CCD photometric observations of stars in the region of NGC 3293 carried out during several observational runs complemented with available information from the literature. Data come then from:

1. The University of Toronto Southern Observatory (UTSO): in two observational runs we obtained $U B V(R I)_{\mathrm{C}}$ photometry using a PM-512 METHACROME UV coated CCD (scale $0^{\prime \prime} .45 /$ pixel covering $4^{\prime}$ on a side) attached to the Hellen-Sawyer $60-\mathrm{cm}$ telescope. The first run was on April 13, 14 and 161994 when three frames were exposed in NGC 3293 using the nitrogen-cooled detector; the second run took place on February 25 and 26 1996, when three more frames were exposed using a glycol-refrigerated detector. Short (2 to $6 \mathrm{~s}$ ), mid (100 to $200 \mathrm{~s}$ ) and long (two series of up to $1100 \mathrm{~s}$ ) exposure times were used to get photometry of the bright stars and to improve the signalnoise-ratio of the faintest stars respectively. A $B V$ comparison frame was taken on February 27, 20' north of the cluster, using similar exposure times. Weather conditions at UTSO were always photometric with seeing values ranging from $1^{\prime \prime} .1$ to $1^{\prime \prime} .5$;

2. The Complejo Astronómico El Leoncito (CASLEO): on April 15 and 17 1999, we made $V(R)_{\mathrm{C}} \mathrm{H}_{\alpha}$ observations in two frames (4'.5 radius) in the area of NGC 3293 using a nitrogen-cooled detector Tek-1024 CCD and focal reducer attached to the $215-\mathrm{cm}$ telescope (scale $0^{\prime \prime} .813 /$ pixel). One frame was centred on the cluster and the other to the west side of it (see Fig. 1). Exposure times in $V, \mathrm{H}_{\alpha}($ on) $=$ $656.6 \mathrm{~nm}$ and $\mathrm{H}_{\alpha}(\mathrm{off})=666.0 \mathrm{~nm}$ filters ranged from $1 \mathrm{~s}$ (in central frame) or $15 \mathrm{~s}$ (west side frame) to $700 \mathrm{~s}$, and from 1 to $150 \mathrm{~s}$ for the $R$ band. In all the cases, mid exposure frames were also taken;

3. The European Southern Observatory (ESO): we complemented our data set with an unpublished $U B V(R I)_{\mathrm{C}} \mathrm{CCD}$ photometric survey conducted by F. Patat and G. Carraro in 1996 with the ESO $0.9 \mathrm{~m}$ Dutch telescope. Details of these observations and data reduction are given in Patat \& Carraro (2001);

4. Other data sources: photometric data for a few bright stars (see Table 1) and available spectral classification were taken from FM80 and TGHH80. Useful complementary information was mainly derived from the Tycho Catalog (ESA 1997) and SIMBAD databases (see Tables 1 and 3).

Figure 1 shows the finding chart of all measured stars. The grey line stands for the area surveyed at UTSO and ESO $\left(U B V(R I)_{\mathrm{C}}\right)$ and the black circle encloses the "cluster region" (see Sect. 3.1).

The reduction process was carried out using IRAF $^{1}$ CCDRED, DAOPHOT and PHOTCAL packages. Instrumental signatures at UTSO and CASLEO frames were removed using bias and dome flat exposures. Dark currents were tested to determine their significance in our observations but they were found negligible. Instrumental magnitudes at $U B V(R I)_{\mathrm{C}} \mathrm{H}_{\alpha}$ were produced via the point spread function, PSF, (Stetson 1987). Calibration sequences in the open clusters Hogg 16 and NGC 5606 (Vázquez et al. 1991, 1994) including several

\footnotetext{
1 IRAF is distributed by NOAO, operated by AURA, under cooperatative agreement with NSF.
} 


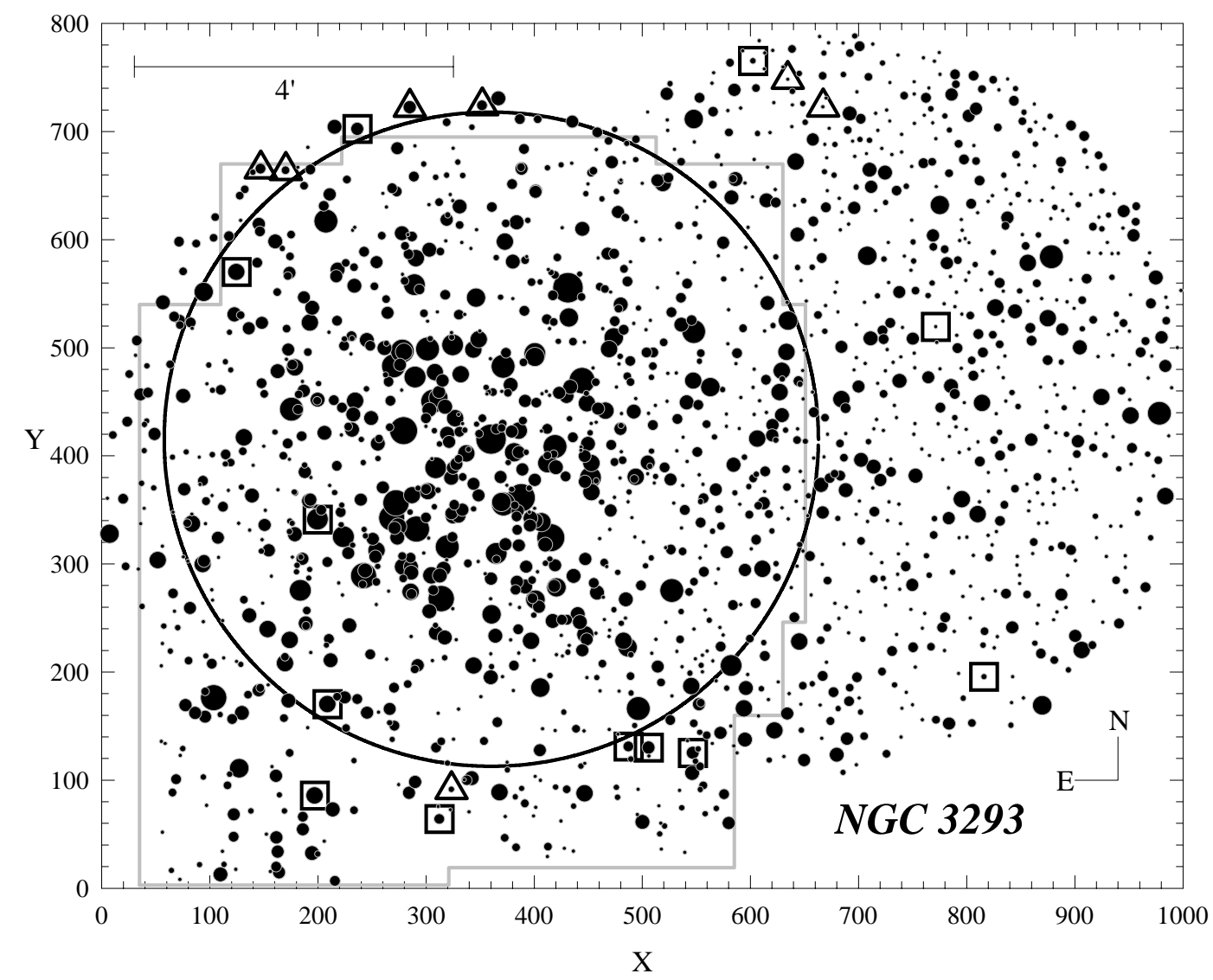

Fig. 1. Finding chart of the observed area ( $V$ filter). Black circle, 4'.1 radius, indicates the adopted limits for the cluster from Sect. 3.1. Grey lines indicates limits of frames from UTSO and ESO observations (that is stars with $U$ and $B$ measurements). For a coordinate reference, star $\# 2(X=360.3 ; Y=415.4)$, adopted as the cluster centre, has $\alpha_{2000}=10^{\mathrm{h}} 35^{\mathrm{m}} 49.3 ; \delta_{2000}=-58^{\circ} 13^{\prime} 27^{\prime \prime} .4$. Stars enclosed by squares and triangles are likely and probable $\mathrm{H}_{\alpha}$ emission stars respectively (see Sect. 5).

Table 1. Photometric catalogue of the NGC 3293 region.

\begin{tabular}{cccccccccccccc}
\hline \hline$\#$ & $\#_{\mathrm{T}}$ & $\#_{\mathrm{FM}}$ & $\#_{\mathrm{F}}$ & $\#_{\mathrm{HM}}$ & $X$ & $Y$ & $V$ & $U-B$ & $B-V$ & $V-R$ & $V-I$ & $\Delta \mathrm{H}_{\alpha}$ & Remarks \\
\hline 1 & 3 & 3 & 3 & - & 431.2 & 555.4 & 6.52 & -0.81 & 0.12 & $0.07_{\mathrm{FM}}$ & $0.09_{\mathrm{FM}}$ &. & $\operatorname{lm} 1-\mathrm{HD} 91943$ \\
2 & 4 & 4 & 4 & - & 360.3 & 415.4 & 6.54 & -0.82 & 0.00 & $0.04_{\mathrm{FM}}$ & $0.09_{\mathrm{FM}}$ &. & $\operatorname{lm} 1-\mathrm{HD} 91969$ \\
$:$ & $:$ & $:$ & $:$ & $:$ & $:$ & $:$ & $:$ & $:$ & $:$ & $:$ & $:$ & $:$ & $:$ \\
1689 & - & - & - & - & 339.9 & 70.4 & $21.65::$ &. & $1.12::$ &. &. &. & - \\
1690 & - & - & - & - & 585.0 & 131.4 & $21.72::$ &. & $0.89::$ &. &. &. & - \\
\hline
\end{tabular}

Comment: Table 1 is available in full in an electronic version at the CDS. A brief summary, only indicating its structure, is shown here.

blue and red stars and standard star groups with blue and red stars from Landolt (1992) were used to produce final colours and magnitudes at UTSO and CASLEO respectively. The final errors of the respective calibration equations, adopted as external errors of our photometry, were $<0.025$. Small mean differences $<0.03$ were found between UTSO and ESO measures. However, the $V(R)_{\mathrm{C}}$ measures made at CASLEO showed a shift relative to UTSO-ESO photometry that was corrected to bring them into the system defined at UTSO-ESO. The estimate of the internal errors was done comparing colour and magnitudes of the stars located in the overlapping zones of our frames. This yields typical differences $<0.03$, up to $V \approx 17$. Table 1 contains the photometric output for 1690 stars; it also includes the star identification, coordinates, the cross-references with other authors and some astronomical catalogues and the membership assignment. Summarising the information available after our survey we have: 1690 stars with $V$ magnitude, 560 stars with $U-B$ index, 940 stars with $B-V, 1550$ with $V-R, 903$ with $V-I$ and 861 with $\mathrm{H}_{\alpha}$ (on) $-\mathrm{H}_{\alpha}$ (off) index.

In relation to our data completeness, we performed an analysis for data from UTSO (Baume 1999) using IRAF tasks ADDSTAR, DAOFIND and ALLSTAR. Then we compared those results with ESO and CASLEO data. That analysis yielded the following completeness results: $100 \%$ down to $V=$ $16,98.7 \%$ down to $V=17,94.3 \%$ down to $V=18$ and $59.2 \%$ down to $V=19$. 
A comparison of our photometry with TGHH80, FM80 and HM82, in the sense "our photometry minus theirs" is shown in Table 2. The influence of (photoelectrically) unresolved stars, binary and variable stars is reflected in the large standard deviations of the mean differences. As shown in the second row, if the known anomalous stars are discarded, the deviations decrease substantially and the mean differences and standard deviations reach acceptable values. The exception is for HM82 photometry where the bulk of their data is photographic.

\section{Data analysis}

\subsection{Cluster angular radius}

To get reliable information on the evolutionary status of an open cluster we have to precisely know its size. In the present case, to address this issue we performed stellar counts in a $30^{\prime} \times 30^{\prime}$ Digitized Sky Survey (DSS) image centred on NGC 3293. All stars detected above a given threshold were assigned $V_{\text {DSS }}$ magnitudes using the DAOPHOT task. $V_{\text {DSS }}$ magnitudes for stars brighter than $V \approx 18$ were next transformed into our photometric system with an accuracy of the order of $\pm 0.5^{\mathrm{m}}$ (a procedure already applied in NGC 6231, Baume et al. 1999). Secondly, assuming the cluster is spherical, the highest apparent star density was fitted with a bi-dimensional Gaussian function. The cluster centre, defined by the highest peak (Battinelli et al. 1991), was found close to star \#2 (No. 4 in FM80 notation) and the angular radius was set at $4^{\prime} .1$, the distance at which the star density equals the background level. A similar procedure but using only our CCD $V$ data, that include stars as faint as $V \approx 20$ (Fig. 1), was applied. We counted stars inside concentric annuli, 100 pixels width, centred on star \#2. As at 650 pixels of star \#2, the annuli are not complete (only small portions of them were observed) we had to extrapolate the counts within each annulus to complete them. The stellar density profile coincides with the background density level at $4^{\prime} .1$ from the cluster centre yielding the same result found with the DSS plates. The black circle of 4'.1 radius shown in Fig. 1 defines then the area occupied by NGC 3293 ("cluster region"). This agrees with TGHH80's finding, that yielded a cluster angular diameter of $10^{\prime}$.

\subsection{Cluster membership}

Proper motions and radial velocity studies yield the most accurate membership determination in a cluster. Proper motions are only feasible for nearby clusters (Sanner \& Geffert 2001) and radial velocities are available mostly for brightest stars. In NGC 3293, 41 of its brightest stars are listed in the Hipparcos/Tycho catalogues (ESA 1997). Only nine of them have parallax and proper motions with relative scientific value. They are indicated in Table 3 although the distance at which NGC 3293 is located makes them, statistically speaking, meaningless to derive distance and memberships. Regarding radial velocities, we used Feast (1958) studies for membership assignment to brightest stars. In particular, star \#3 (No. 21 in FM80 notation) is a red super-giant (CPD - 57 3502;
IRAS source 10338-5759) and is a likely cluster member according to its radial velocity value and its spectrophotometric distance modulus (see Table 3).

In the classical photometric method to address cluster memberships (e.g. Baume et al. 1999), the consistency of the location of each star is assessed simultaneously in all the photometric diagrams (the two colour diagram, TCD, and the different CMDs). Other authors use reddening limits within which cluster members should lie or they adopt maximum departures from a reference line as the ZAMS (Deeg \& Ninkov 1996; Hillenbrand et al. 1993). Our method works well for bright members, but it becomes unpractical to detect members among faint stars in crowded fields. It was mentioned by Abt (1979) that the classical method is controversial; however, as was stated by TGHH80 and emphasised by Forbes (1996), it is good enough when it relies on a careful inspection of the TCD and consistent reddening solutions are applied. The method works well too for nearby, not much reddened, intermediate and old clusters without traces of contracting stars (Stahler \& Fletcher 1991), but it fails for young clusters where contracting stars and field interlopers very often occupy the same location on the CMD. If the reddening is high and the cluster is distant, the results are clearly dubious. Therefore, in order to minimise this problem, we divided our data set in two groups, stars brighter than $V \approx 14$ and fainter than that value.

\subsubsection{Bright cluster members}

For stars brighter than $V \approx 14$, the method described above was combined with spectroscopic data (whenever possible) to classify stars as likely members ( $(\mathrm{m} 1)$ and probable members (pm1), which are indicated with black filled symbols in our figures. The following main features can be outlined from inspection of the cluster photometric diagrams:

- The TCD (Fig. 2a) shows, down to $B-V \approx 0.3$, a well recognisable blue and scattered main sequence composed of stars with spectral types earlier than A0 mostly included inside reddening values $0.20<E_{B-V}<0.45$ (see Sect. 4.1);

- The four CMDs (Fig. 3), in turn, show a clear and wellpopulated upper main sequence slightly widened because of differential reddening. The brightest stars of the cluster main sequence are mostly placed above the ZAMS (Schmidt-Kaler 1982) while stars with $V>13$ are on the ZAMS;

- None of the diagrams show evidence of strong contamination of field stars among bright members. Actually, most of field stars start mixing with cluster members below $V \approx 12$, becoming an important obstacle in analysing the faint part of the cluster.

\subsubsection{Faint cluster members}

Determining membership for stars with $V>14$ requires a different procedure. If $V$ vs. $B-V$ and/or $V$ vs. $V-R$ CMDs are available for the "cluster region" and a "field region", we can subdivide them into a grid of boxes $(\Delta V=1 \times$ $\Delta(B-V)=0.1$ and $\Delta V=1 \times \Delta(V-R)=0.1)$ and build 
Table 2. Differences with previous photometric works in the sense "our photometry minus other authors".

\begin{tabular}{l|rrrcrc}
\hline \hline Work & \multicolumn{1}{|c}{$\Delta(V)$} & \multicolumn{1}{c}{$\Delta(U-B)$} & \multicolumn{1}{c}{$\Delta(B-V)$} & $\Delta(V-R)$ & $\Delta(V-I)$ & $N$ \\
\hline FM80 & $-0.06 \pm 0.21$ & $-0.01 \pm 0.19$ & $0.02 \pm 0.04$ & $-0.03 \pm 0.05$ & $-0.04 \pm 0.07$ & $(35)$ \\
& $-0.01 \pm 0.03$ & $0.03 \pm 0.05$ & $0.03 \pm 0.03$ & $-0.03 \pm 0.03$ & $-0.03 \pm 0.06$ & \\
\hline TGHH80 & $-0.01 \pm 0.10$ & $0.01 \pm 0.11$ & $-0.00 \pm 0.09$ & & & $(86)$ \\
& $-0.01 \pm 0.06$ & $0.01 \pm 0.08$ & $-0.00 \pm 0.04$ & & & $(278)$ \\
\hline HM82 & $0.04 \pm 0.16$ & $0.01 \pm 0.16$ & $0.00 \pm 0.12$ & & & \\
\hline
\end{tabular}
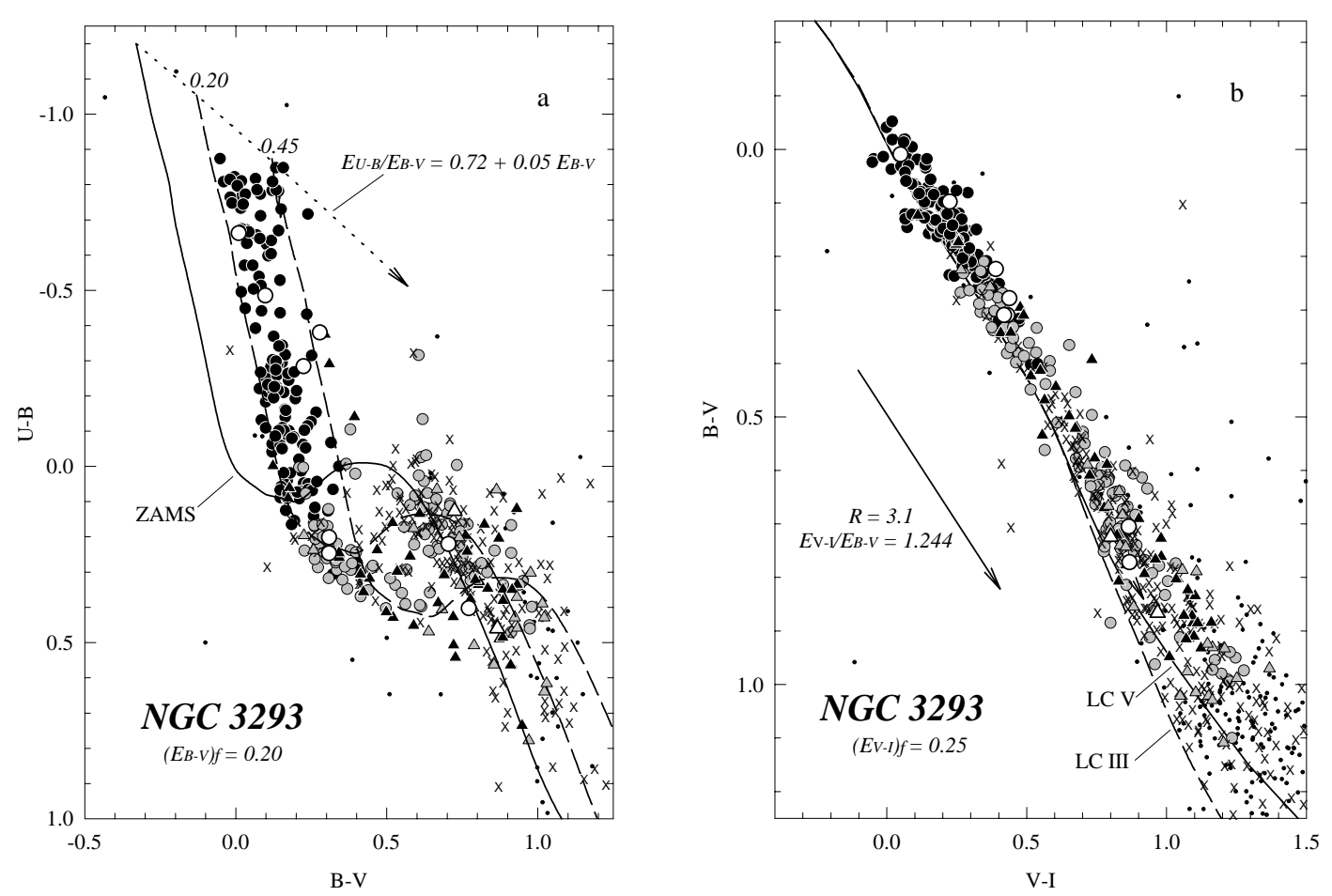

Fig. 2. a) Two colour diagram (TCD). Symbols have the following meaning: circles are likely member stars (lm) and triangles are probable member stars $(\mathrm{pm})$; black symbols indicate membership obtained from the classical photometric method and grey ones those obtained from the subtraction method (see Sect. 3.2 for explanation of the methods); crosses are non-member stars (nm) and dots are stars without membership assignment; white symbols indicate likely and probable $\mathrm{H}_{\alpha}$ emission stars (circles and triangles respectively). Solid line is the Schmidt-Kaler (1982) ZAMS; dashed lines indicate the position of the ZAMS shifted by $E_{B-V}=0.20$ and 0.45 . Dotted arrow indicates the normal reddening path. b) $B-V$ vs. $V-I$ diagram. Symbols as in Fig. 2a. Solid and dashed lines are the intrinsic positions for stars of luminosity classes $\mathrm{V}$ and III respectively (Cousins 1978). Solid arrow gives the normal reddening path for $R=3.1$.

two-dimensional histograms for each CMD. Subtracting the respective two-dimensional histograms (this mean "cluster region" - "field region"), we can remove (statistically speaking) the contamination produced by field stars onto the CMDs of the "cluster region". This way, counts left on the resulting two-dimensional histogram define the locus occupied by cluster member stars on the CMDs (Chen et al. 1998). Naturally, the reliability of the number of members obtained and the locus they occupy depend on two factors: a) the adopted "field region" must be representative of the field star distribution over the "cluster region" and b) the "cluster region" must include the whole extension of the cluster to account for mass segregation. Actually, the true cluster size will strongly depend on the extension of the segregation process outward of it. If the "field region" is too close to the cluster, it may contain low mass segregated members; if it is far from the cluster it may not represent the true field star distribution across it. Finally, dust clouds and emission nebulae (very frequent in young clusters near the galactic plane) along with differential reddening lead to wrong estimations of the field star distribution, too (Mermilliod 1976; Prisinzano et al. 2001; see Forbes 1996 for details).

In the remainder of this section, all procedures will be applied only to stars in the range $14<V<18$ with photometric errors $<0.1$. A first point to treat is whether comparison fields around NGC 3293 show any strong differences produced by random stellar fluctuations and/or the type of stellar data. To ease the analysis we divided our data set into four groups:

- Sample I: $V, B-V$ and $V-R$ data from stars placed in the cluster area, up to a $4^{\prime} .1$ radius. This sample is the "cluster region" and contains cluster stars plus field stars;

- Sample II: $V, B-V$ and $V-R$ data for stars placed between $4^{\prime} .1$ radius and the limits of the grey line squared frames shown in Fig. 1; 

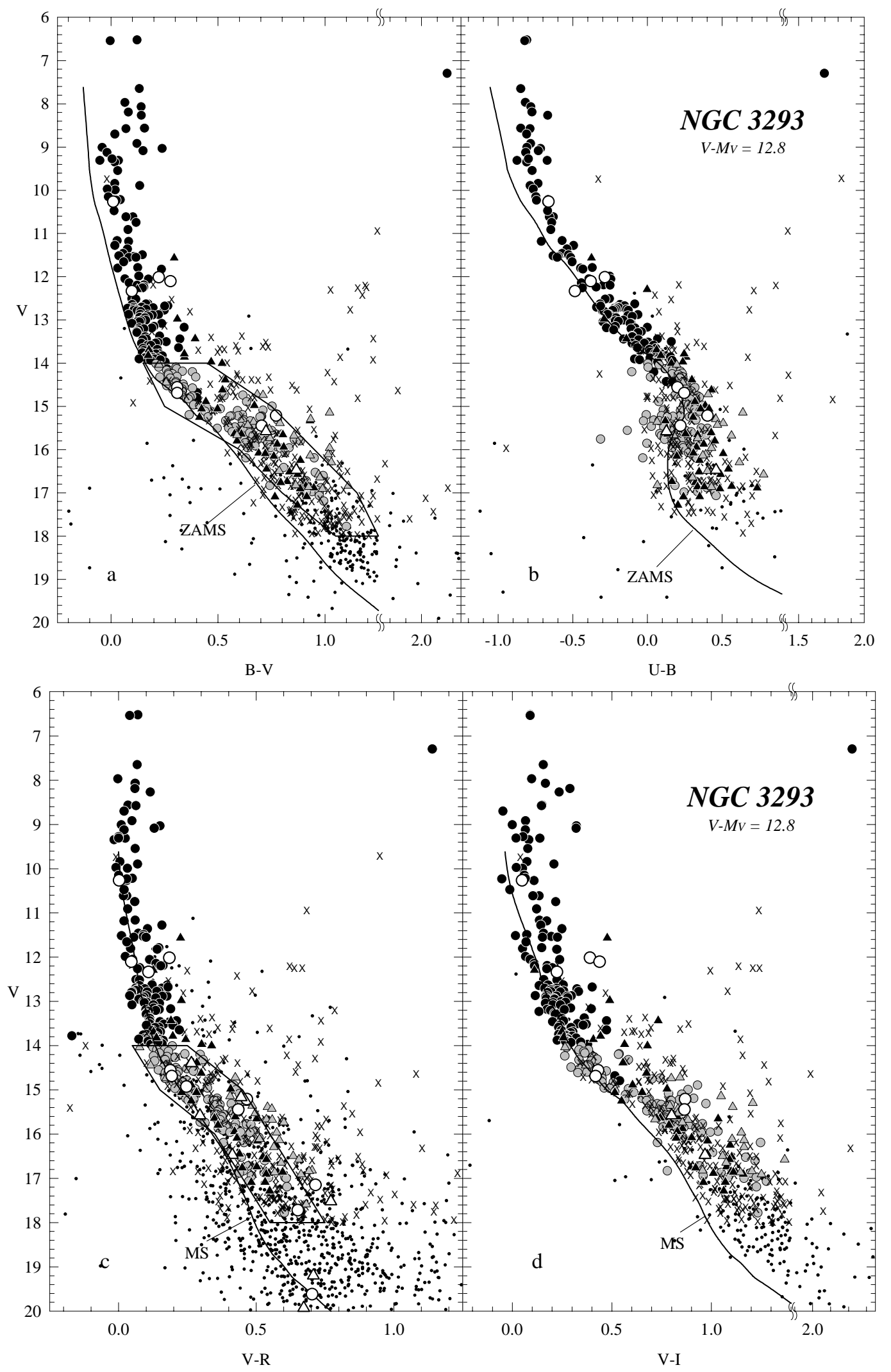

Fig. 3. Colour-magnitude diagrams (CMDs). Symbols as in Fig. 2a. Solid line in a) and b) is the Schmidt-Kaler (1982) ZAMS and the Cousins' (1978) intrinsic line for luminosity class $V$ stars in c) and d) fitted to the apparent distance modulus $V-M_{V}=12.8\left(V-M_{V}=V_{0}-M_{V}+R\left(E_{B-V}\right)_{\mathrm{f}}\right.$, see Sects. 4.1 and 4.2). Closed curves in a) and c) indicate limits adopted for faint star membership (see Sect. 3.2.2). 


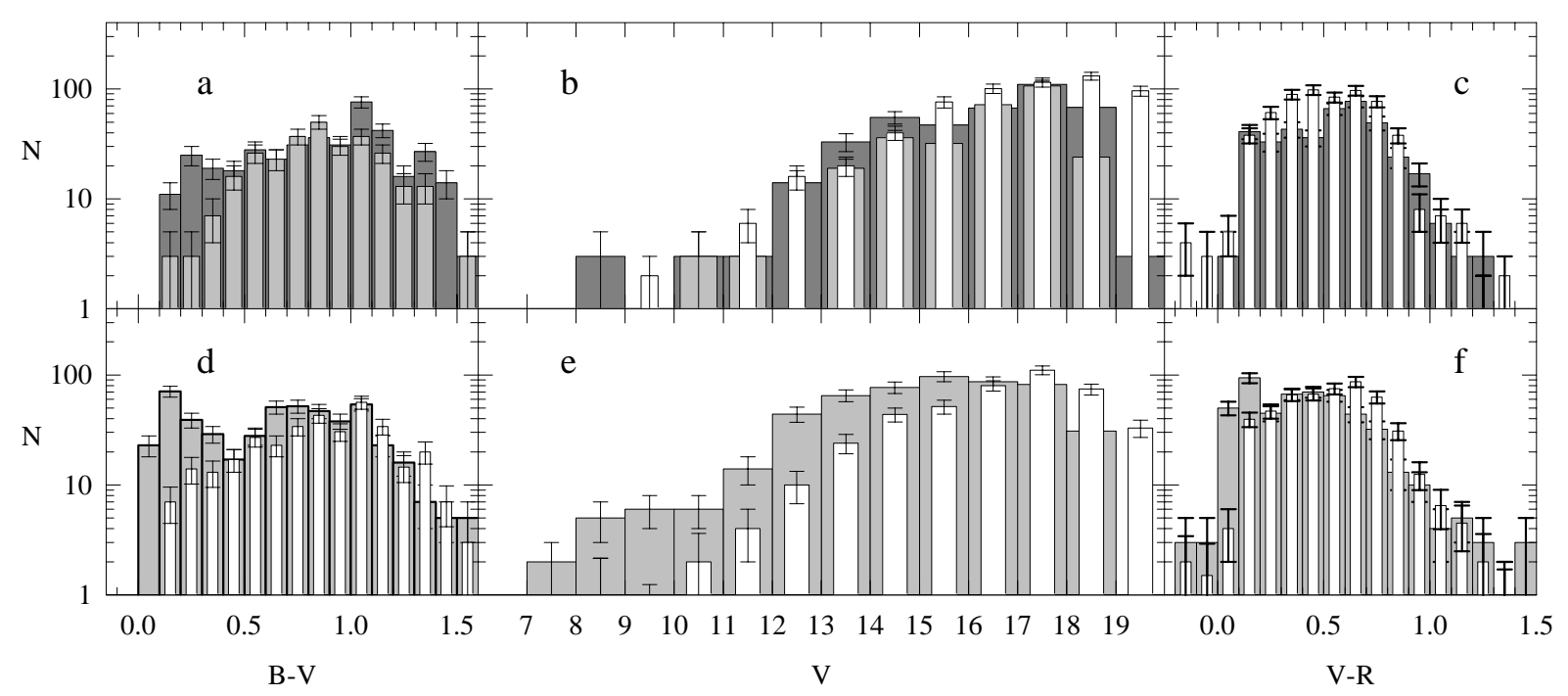

Fig. 4. Marginal distributions of the two-dimensional histograms of the different samples taken from our data (see Sect. 3.2.2). Upper panels, a)-c), represent what we call "field regions": dark-grey distributions belong to Sample II, grey ones to Sample III and white ones to Sample IV. In d)-f), grey distributions belong to Sample I ("cluster region" containing cluster plus field stars) while the white ones stand now for the average of samples $I I-I V$ (our best representation of the "field region").

- Sample III: $V$ and $B-V$ data from stars placed in the comparison field (see Sect. 2) 20' north of the cluster;

- Sample IV: $V$ and $V-R$ data for stars from the grey line squared frames outwards.

We warn that samples $I I-I V$ are different representations of what we called the "field region". As they cover different areas they were adequately scaled to the "cluster region" area to build the two-dimensional histograms of each sample. Any strong spatial variation among the field samples should be revealed by the marginal distributions of the two-dimensional histograms of each sample. A brief inspection of them, shown in Fig. 4, shows that:

- Figures $4 \mathrm{~b}$ and $4 \mathrm{e}$ show the same degree of completeness of the photometry in the "cluster region" and the field samples down to $V \approx 18$;

- Figures $4 \mathrm{a}-\mathrm{c}$ show that different field samples have not only the same shape (down to $V \approx 18$ ) but also approximately the same number of stars. Aside from uncertainties produced by small number statistics, the actual true distribution of field stars over the cluster surface should not differ too much from these three.

To quantify the last issue, we applied a Kolmogorov-Smirnoff test to the field distributions confirming that, for $V<18$, the three field samples are similar at the level $\alpha=0.05$. Thus, the average of their respective two-dimensional histograms ( $V$ vs. $B-V$ and/or $V$ vs. $V-R$ ) yields the best representation of the "field region" across NGC 3293. The corresponding marginal distributions are presented as white histograms in Figs. 4d-f.

Another point to analyse is whether NGC 3293 has undergone mass segregation and shows a core/halo structure, as suggested by HM82. Although in Sect. 3.1 we did not find evidence of any halo around NGC 3293, we compared the ratios of stellar densities found in the "cluster region" $\left(\rho_{\mathrm{IN}}\right)$ and outside it $\left(\rho_{\text {OUT }}\right)$ as a function of $V$. If an appreciable number of less

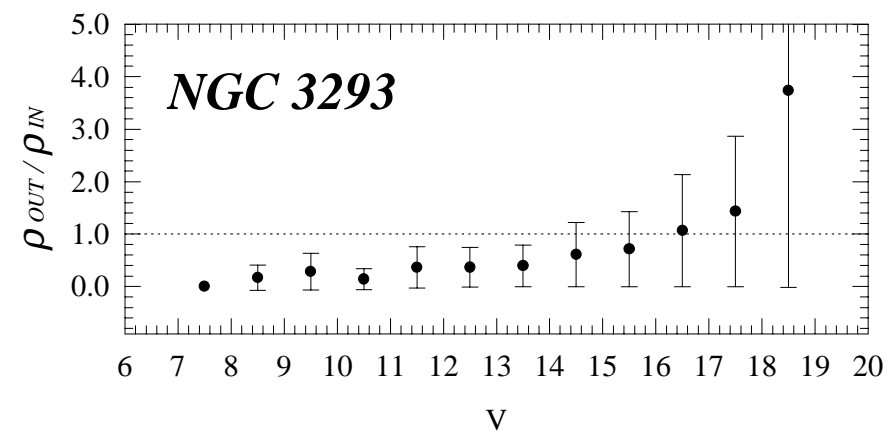

Fig. 5. $\rho_{\text {OUT }} / \rho_{\text {IN }}$ vs. $V$ diagram. Poisson statistic error in each magnitude are also indicated.

massive (faint) stars were located outside the cluster boundaries due to an active mass segregation process, then the $\rho_{\text {OUT }} / \rho_{\text {IN }}$ ratio should show that. As expected, the plots of Fig. 5 do not indicate any appreciable star excess outside the cluster area, but just a very slow increase at very faint magnitudes (at the level where the completeness starts to be questionable). We conclude that the cluster has not undergone mass segregation and its limits from Sect. 3.1 are fully reliable.

We proceeded then to subtract the average twodimensional histogram of the "field region" from the one of the "cluster region", obtaining the contamination-free $V$ vs. $B-V$ and $V$ vs. $V-R$ distributions. They revealed the presence of stellar bands above the ZAMS with confident lower and upper limits. Such limits were used on the CMDs (see Figs. 3a and $3 \mathrm{c}$ ) to define which stars are (from a statistical point of view) cluster members following the next criteria:

- A star is a cluster likely member $(\operatorname{lm} 2)$ if it is found inside the cluster boundaries defined in Sect. 3.1 and is simultaneously included inside the band limits in the two CMDs $(V$ vs. $B-V$ and $V$ vs. $V-R$ ); 
- A star is a probable member (pm2) if, apart from being spatially well located, it fits, at least, in any of the high density zones defined in the CMDs and shows slight departures in the other.

Finally, the resulting sample was filtered (following a random distribution) in order to obtain final CMDs with a number of stars in each colour-magnitude box similar to that obtained after the subtraction of the two-dimensional histograms. The adopted memberships are shown with grey filled symbols in the photometric diagrams of our figures. Notice in advance that member stars with $V>15.5$ are mainly placed above the ZAMS line in the $V$ vs. $B-V$ and $V$ vs. $V-I$ diagrams (we will return to this point in Sects. 4.3 and 6).

\section{Cluster parameters}

\subsection{Corrected colours and magnitudes of cluster members}

For the estimation of the cluster mean colour excesses, we used first the 14 stars with known spectral classification and luminosity class IV-V (see Table 3) using the Schmidt-Kaler (1982) relations of spectral types and intrinsic colours (Be stars were excluded). The plot of excesses is shown in Fig. 6a together with the standard $E_{U-B} / E_{B-V}$ relation. The fit is poor probably because of colour anomalies of the many variable stars present in the sample. Mean value excesses of these stars were $E_{B-V}=0.29 \pm 0.06$ (s.d.) and $E_{U-B}=0.23 \pm 0.08$ (s.d.). Secondly, we applied the well-known relations $E_{U-B} / E_{B-V}=$ $0.72+0.05 E_{B-V}$ and $(U-B)_{0}=3.69(B-V)_{0}+0.03$ to get the intrinsic colours of those stars with $V<14$ adopted as likely members and without spectral classification (mostly located at $0.20<E_{B-V}<0.45$, see Fig. 2a). When we included this last group of stars in the mean colour excesses computations, the obtained values were $E_{B-V}=0.29 \pm 0.06$ (s.d.) and $E_{U-B}=0.21 \pm 0.05$ (s.d.), almost identical to those obtained first. We de-reddened cluster members with $V<14$ (except star \#3) using individual excesses; meanwhile other cluster members and star \#3 were de-reddened using these last mean excess values. The estimated foreground colour excesses of NGC 3293 were $\left(E_{B-V}\right)_{\mathrm{f}}=0.20$ and $\left(E_{U-B}\right)_{\mathrm{f}}=0.15$, slightly lower than the typical ones for southern Carina ( $\operatorname{Tr} 15$, Carraro 2002; Tr 14/15/16, Tapia et al. 2002).

The next step was to know the absorption law valid in NGC 3293 given by $R=A_{V} / E_{B-V}$. Galactic regions with normal absorption have a mean of $R=3.1-3.2$ although larger $R$-values are especially found in regions of recent star formation. To compute the local $R$-value we obtained individual $E_{B-V}, E_{V-R}$ and $E_{V-I}$ excesses through the $(B-V)_{0}$ with $(V-R)_{0}$ and $(V-I)_{0}$ relations for stars without spectral classification (circles in Figs. 6b,c) and the relation between MK types and $(V-R)_{0}$ and $(V-I)_{0}$ for stars with spectral types (squares in Figs. 6b,c), both from Cousins (1978). $E_{V-R} / E_{B-V}$ and $E_{V-I} / E_{B-V}$ ratios depend on the $R_{V}$-values as when $E_{V-R} / E_{B-V}=0.57$ and $E_{V-I} / E_{B-V}=1.244$ the interstellar material is normal (Vázquez et al. 1995; Dean et al. 1978). The plot of 140 stars with $E_{B-V}, E_{V-R}$ and $E_{V-I}$ are depicted in Figs. $6 \mathrm{~b}$ and $6 \mathrm{c}$ showing an excellent agreement

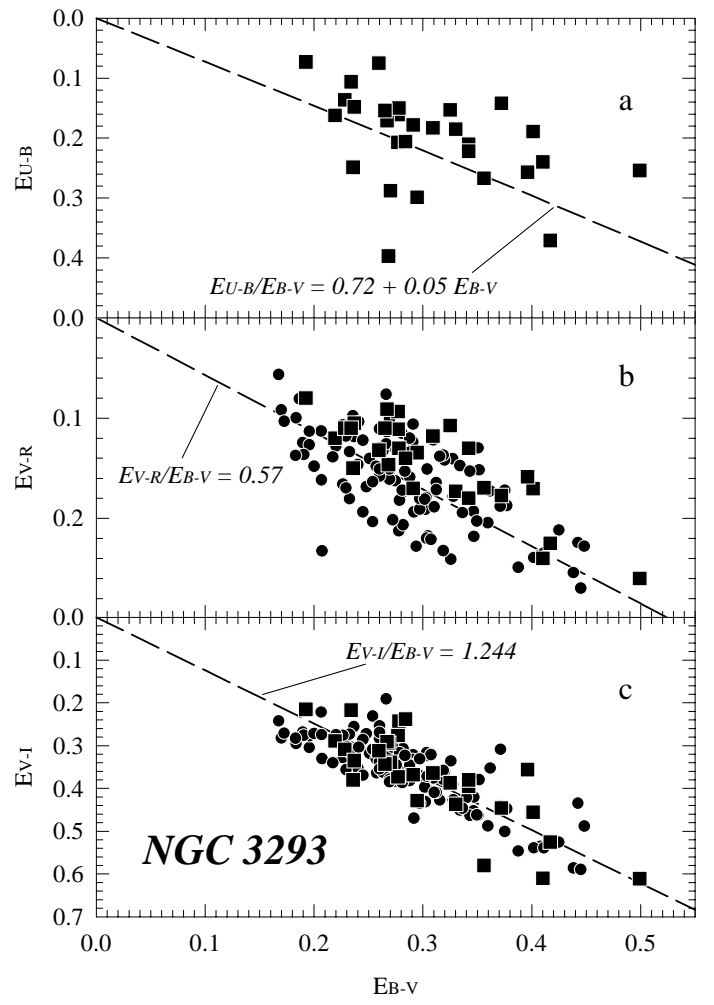

Fig. 6. $E_{U-B}, E_{V-R}$ and $E_{V-I}$ vs. $E_{B-V}$ diagrams for likely cluster members with $V<14$ except star \#3 and Be stars. Squares are stars with available spectral classification and dashed curves indicate normal relations.

with the typical reddening relations. The mean ratios found are $E_{V-R} / E_{B-V}=0.54 \pm 0.15$ (s.d.) and $E_{V-I} / E_{B-V}=$ $1.26 \pm 0.18$ (s.d.), indicative of a normal reddening law for which is $R=3.1$. The foreground excesses we found were $\left(E_{V-I}\right)_{\mathrm{f}}=0.25$ and $\left(E_{V-R}\right)_{\mathrm{f}}=0.11$. Finally we mention that FM80 claim that $R$ can increase to 3.5 . That is marginally probable seeing the plots of Figs. 6b,c, although the scatter around the mean lines may be mainly caused by circumstellar envelopes, variability and/or binarity instead of anomalies in the absorption. Therefore, we adopted $R=3.1$ to obtain corrected magnitudes as $V_{0}=V-3.1 E_{B-V}$.

\subsection{Cluster distance and size}

The distance of NGC 3293 was derived superposing the Schmidt-Kaler (1982) ZAMS onto the reddening-free CMD. The best ZAMS fitting was achieved for a distance modulus $V_{0}-M_{V}=12.2 \pm 0.2$ (error from eye-inspection). We also applied the spectroscopic parallax method to 33 stars to get the cluster distance modulus (using the relation of spectral types and $M_{V}$ from Schmidt-Kaler 1982) that yielded $12.1 \pm 0.5$. If only 17 stars of luminosity class IV-V are used, the distance modulus turns out to be $12.3 \pm 0.5$ (see last column in Table 3 ). The large dispersions of the moduli associated with the spectroscopic parallax method may be intrinsic for early type stars (Conti \& Underhill 1988) or produced by a high percentage of variable and binary stars (Feast 1958). Our distance modulus is a bit larger than the ones given by Feast (1958), $12.08 \pm 0.1$, 
Table 3. Main characteristics of bright stars in NGC 3293.

\begin{tabular}{|c|c|c|c|c|c|c|c|c|c|c|}
\hline$\#$ & $\#_{\mathrm{FM}}$ & $S T$ & & Remarl & & $E_{B-V}$ & $E_{U-B}$ & $E_{V-R}$ & $E_{V-I}$ & $V_{0}-M_{V}$ \\
\hline 1 & 3 & B0..5 Iab & V513 Car & - Hip-Тyc & - & 0.34 & 0.22 & 0.18 & 0.38 & 11.86 \\
\hline 2 & 4 & B0 Ib & & - Нip-Тус & - & 0.24 & 0.25 & 0.15 & 0.38 & 11.91 \\
\hline 3 & 21 & M1.5 Iab & V361 Car & - IRAS & - & & & & & 12.00 \\
\hline 4 & 22 & B1 II & & - & - s.d.s. & 0.37 & 0.14 & 0.18 & 0.45 & 11.90 \\
\hline 5 & 20 & B1 III & V439 Car & - & - & 0.33 & 0.15 & 0.11 & 0.39 & 11.36 \\
\hline 6 & 25 & B1 III & & - & - & 0.40 & 0.19 & 0.17 & 0.46 & 11.23 \\
\hline 7 & 6 & B0.5 III & & - & - & 0.36 & 0.27 & 0.17 & 0.58 & 11.84 \\
\hline 8 & 8 & B0.5 III & & - Нip-Тус & - & 0.42 & 0.37 & 0.23 & 0.53 & 11.72 \\
\hline 9 & 26 & B1 III & V379 Car & - & $-\mathrm{Be}^{(3)}-\beta \mathrm{Ceph}$ & 0.42 & 0.12 & 0.14 & 0.44 & 11.67 \\
\hline 10 & 7 & B1 III & & - & - b.s. ${ }^{(1)}$ & 0.33 & 0.19 & 0.17 & 0.44 & 11.95 \\
\hline 11 & 16 & B1 IV & V403 Car & - & $-\beta \mathrm{Ceph}$ & 0.28 & 0.15 & 0.13 & 0.24 & 11.44 \\
\hline 12 & 27 & B0.5 III & V380 Car & - & - $\beta$ Ceph & 0.40 & 0.26 & 0.16 & 0.36 & 12.44 \\
\hline 13 & 5 & B1 III & V381 Car & - & - $\beta$ Ceph - b.s. ${ }^{(1)}$ & 0.22 & 0.16 & 0.12 & 0.29 & 12.73 \\
\hline 14 & 19 & B1 III & & - & - s.d.s. & 0.50 & 0.25 & 0.26 & 0.61 & 11.89 \\
\hline 15 & 2 & B1 III & & - Нур-Тус & - & 0.41 & 0.24 & 0.24 & 0.61 & 12.22 \\
\hline 17 & 18 & B1 V & V406 Car & - & - $\beta$ Ceph & 0.27 & 0.15 & 0.11 & 0.34 & 11.65 \\
\hline 18 & 14 & B $0.5 \mathrm{~V}$ & V405 Car & - & - $\beta$ Ceph & 0.23 & 0.14 & 0.11 & 0.31 & 12.20 \\
\hline 19 & 24 & B1 III & V378 Car & - & - $\beta$ Ceph & 0.29 & 0.30 & 0.13 & 0.43 & 12.80 \\
\hline 20 & 23 & B1 III & V404 Car & - & - $\beta$ Ceph - s.d.s. & 0.28 & 0.16 & 0.09 & 0.37 & 12.89 \\
\hline 21 & 10 & B1 V & V401 Car & - & - $\beta$ Cерh & 0.29 & 0.18 & 0.17 & 0.37 & 11.84 \\
\hline 22 & 43 & K5 & & - Нip-Тус & - r.fg.s. & & & & & \\
\hline 23 & 42 & A0 & & - Нip-Тус & - & & & & & \\
\hline 25 & 12 & B1 V & V402 Car & - & $-\mathrm{Be}^{(2)}-$ b.s..$^{(1)}$ & 0.39 & 0.16 & 0.18 & 0.50 & 12.77 \\
\hline 27 & 9 & $\mathrm{~B} 2 \mathrm{~V}$ & & - Нip-Тус & - b.s..$^{(1)}$ & 0.26 & 0.07 & 0.13 & 0.31 & 11.59 \\
\hline 28 & 13 & $\mathrm{~B} 1.5 \mathrm{~V}$ & & - & - & 0.24 & 0.15 & 0.10 & 0.34 & 12.22 \\
\hline 30 & 15 & $\mathrm{~B} 1 \mathrm{~V}$ & & - & - s.d.s. & 0.28 & 0.21 & 0.14 & 0.24 & 12.55 \\
\hline 31 & 28 & B1 V & & - & $-\mathrm{H}_{\alpha}-$ b.s. ${ }^{(1)}$ & 0.27 & 0.29 & 0.11 & 0.34 & 12.63 \\
\hline 33 & 17 & $\mathrm{~B} 2.5 \mathrm{~V}$ & V440 Car & - & - & 0.23 & 0.11 & 0.11 & 0.22 & 11.75 \\
\hline 34 & 33 & B2 V & & - & - & 0.34 & 0.21 & 0.13 & 0.40 & 11.95 \\
\hline 35 & 29 & B2 V & & - & - & 0.31 & 0.18 & 0.12 & 0.36 & 12.06 \\
\hline 38 & - & B8 & & - Hip-Тyc & - b.bg.s. & & & & & \\
\hline 47 & 31 & $\mathrm{~B} 2 \mathrm{~V}$ & & - & - & 0.28 & 0.21 & 0.11 & 0.28 & 13.06 \\
\hline 48 & - & & & - Hip-Тyc & - & & & & & \\
\hline 96 & 30 & B5 V & & - & - & 0.27 & 0.40 & 0.15 & 0.29 & 13.01 \\
\hline 115 & 35 & B7 V & & - & - & 0.27 & 0.17 & 0.09 & 0.29 & 12.64 \\
\hline 116 & 34 & B8 V & & - & - & 0.19 & 0.07 & 0.08 & 0.22 & 12.47 \\
\hline 120 & 32 & $\mathrm{~B} 8 \mathrm{~V}$ & & - & $-\mathrm{Be}^{(1)}$ & 0.19 & 0.21 & 0.13 & 0.27 & 12.50 \\
\hline
\end{tabular}

\section{Remarks:}

b.s. = binary star.

s.d.s. $=$ star in double system.

r.fg.s. = red foreground star.

b.bg.s. = blue background star. $\beta$ Ceph $=$ beta cepheid star.

$\mathrm{H}_{\alpha}=$ likely $\mathrm{H}_{\alpha}$ emission star.

$\mathrm{Be}=\mathrm{Be}$ star.

IRAS = Source IRAS 10338-5756.

Hip-Tyc $=$ Star with paralax and proper motion measurements

(see Table 1 for HIP/TYC identification).

(1) = Feast (1958); (2) = Schild (1970); (3) = Shobbrook (1980).

FM80, $12.1 \pm 0.15$, TGHH80 11.99 \pm 0.13 , Shobbrook (1983), $11.95 \pm 0.1$ and smaller than the one from Balona \& Crampton (1974), 12.32 \pm 0.09 . Nevertheless, as at $1 \sigma$, all of them are almost coincident, we adopted $V_{0}-M_{V}=12.2 \pm 0.2$ which yields a cluster distance $d=2750 \pm 250 \mathrm{pc}$. The absolute magnitude scale $M_{V}$ shown in Fig. 7 was set with this mean value.

When the above distance modulus is combined with the angular radius obtained in Sect. 3.1 it yields a linear cluster radius $3.3 \pm 0.3 \mathrm{pc}$, comparable to the sizes of other studied young open clusters such as $\mathrm{Cr} 272,4.0$ pc; NGC 6231, 4.1 pc, and H-M 1, 2.9 pc (from Vázquez et al. 1997; Baume et al. 1999 and Vázquez \& Baume 2001 respectively). This result however disagrees with the Janes et al. (1988) result that most young clusters have diameters less than $5 \mathrm{pc}$.

\subsection{Cluster age}

\subsubsection{Nuclear age}

Regarding the nuclear age of NGC 3293, the isochrones derived from Girardi et al. (2000) evolutionary models (computed with solar metallicity, mass loss and overshooting) are shown 

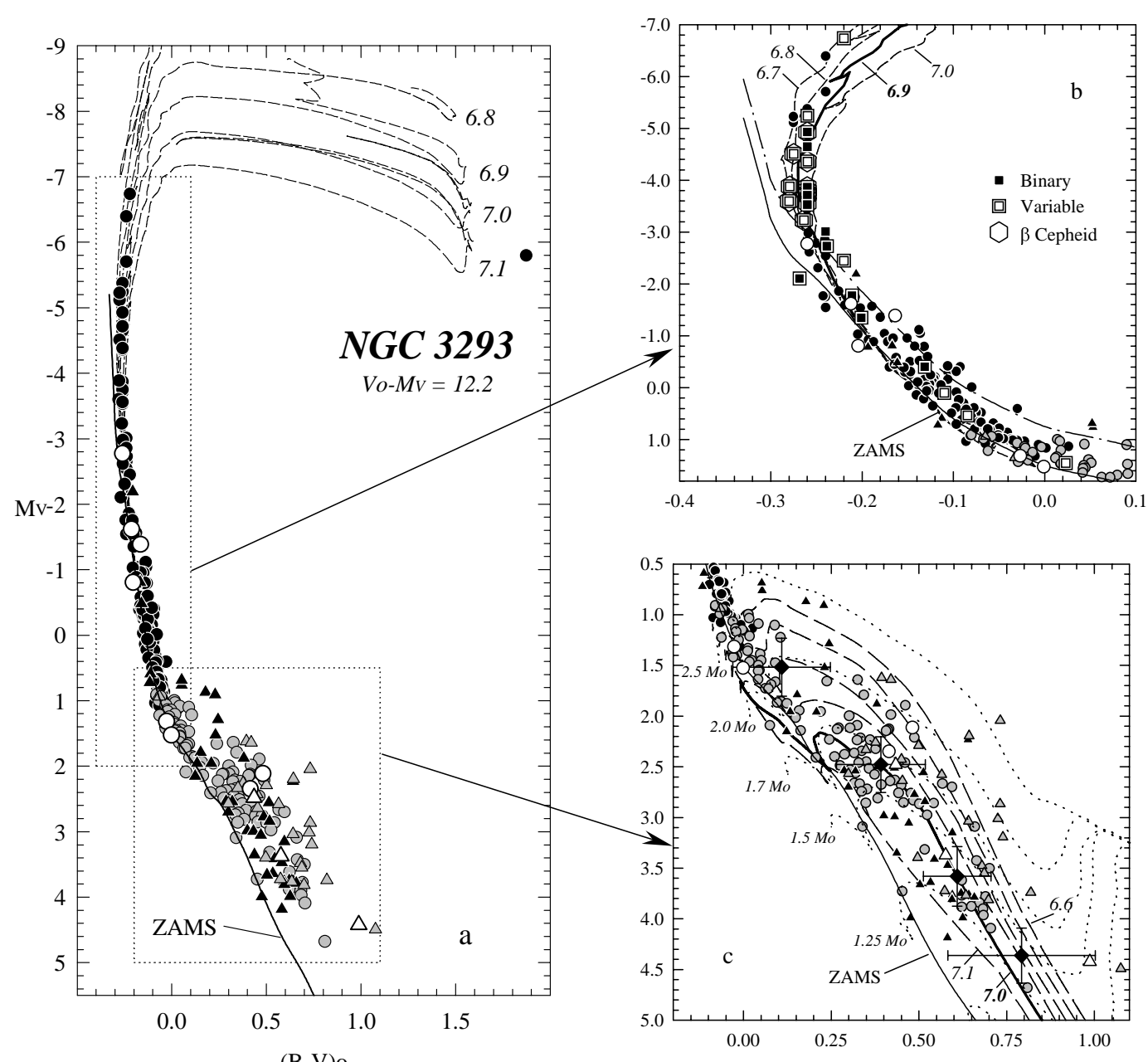

Fig. 7. a) The entire $M_{V}$ vs. $(B-V)_{0}$ diagram. Symbols as in Fig. 2a. Solid line is the Schmidt-Kaler (1982) ZAMS. Dashed lines are the isochrones from Girardi et al. (2000). Numbers give the $\log$ age. b) An enlargement of the upper main sequence showing the binary envelope (dotted - dashed line) $0.75^{\mathrm{m}}$ above the ZAMS. The thick line is the isochrone of 8 Myr. Numbers as in a). c) An enlargement showing the faint sequence of the cluster. Dotted and dashed lines represent the tracks and isochrones (Bernasconi \& Maeder 1996). Numbers indicate the masses of each track and the $\log ($ age $)$. The $10 \mathrm{Myr}$ isochrone is shown as a thick line. Mean values and dispersions of PMS stars grouped at intervals $\Delta M_{V}=1$ are also indicated.

in Figs. 7a,b superposed on the cluster upper main sequence. Because an important fraction of bright members were dereddened using their spectral types, some scatter arises that precludes a single isochrone fitting the bright part of NGC 3293; binaries and fast rotators are likely to be another source of scatter in upper CMD. The envelope of binaries $0.75^{\mathrm{m}}$ above the ZAMS encloses quite well most of the binary stars in the upper main sequence. We draw attention onto the red super-giant (star \#3) which is not included by any isochrone (Fig. 7a), but it lies close to the isochrones of 10-12.6 Myr. As many other red-supergiant stars, this star is not contained by any isochrone, a frequent effect already reported in Meynet et al. (1993).

From stars with $M_{V}<-4$, the nuclear age of NGC 3293 goes from 6.5 to $10.0 \mathrm{Myr}$ with a probable mean age of $8 \mathrm{Myr}$. The cluster is thus older than in previous investigations, $5 \pm$ 2 Myr by TGHH80 and 6 \pm 2 Myr from HM82. Such differences may have their origin in different (smaller) distance moduli estimates and also in the use of different isochrone sets.

\subsubsection{Contraction age}

As indicated in Sect. 3.2.2, a relevant feature emerging from the cluster lower sequence (Fig. 7c) is that the left envelope of NGC 3293 does not follow the shape of the ZAMS. The lower cluster sequence shows a bend at $M_{V} \approx+2$, below of which it lies $1^{\mathrm{m}}$ above the ZAMS, approximately constituting a parallel sequence that confirms the earlier assertion of HM82 that faint stars are mostly above the ZAMS.

As contamination of field interlopers already has been removed, the stars in this parallel sequence have to be interpreted as stars in contraction phase towards the ZAMS. The strong $M_{V}$ scatter at constant colour is a normal feature associated with the age spread (Iben \& Talbot 1966) among these type of objects (cf. Fig. 3 in Preibisch \& Zinnecker 1999). However, the contrast between the wide PMS band and the sharp upper main sequence in Fig. 7c is produced primarily by: a) differential reddening that was not removed from the main sequence faint stars (they were de-reddened using the mean excess values derived 
in Sect. 4.1) and, b) several intrinsic factors detailed below. In overlaying in Fig. 7c the accretion evolutionary models developed by Bernasconi \& Maeder (1996) we find that the PMS objects have masses ranging from 1 to $2.5 \mathcal{M}_{\odot}$ and ages from 6 to $12 \mathrm{Myr}$, a PMS age range close to $\sim 5 \mathrm{Myr}$ found recently in the other Carina clusters $\operatorname{Tr} 14, \operatorname{Tr} 15$ and $\operatorname{Tr} 16$ by Tapia et al. (2002) and confirmed in Tr 15 by Carraro (2002).

We do not find a single isochrone that fits the whole lower sequence; indeed PMS stars tend to cross several of them. This lack of alignment led Iben \& Talbot (1966) to reject out the hypothesis of coeval star formation in the clusters NGC 2264 and NGC 6530. Recently, the same was done in NGC 2264 by Flaccomio et al. (1999). Notwithstanding, departures from a single isochrone produced by differential reddening, binarity/multiplicity, random distribution of accretion discs around single and multiple systems, photometric errors and physical uncertainties in the evolutionary models are expected to happen. The influence of differential reddening is hard to estimate as it affects different stars in different manners. As for binarity, it is very frequent not only among normal less massive stars (>50\%, Bessell \& Stringfellow 1993; Preibisch \& Zinnecker 1999) but also among PMS stars (Hartigan et al. 1994). Binarity raises the stars above a reference line (e.g. the ZAMS) in a way that depends on the mass ratio of the binaries. As for the accretion stellar discs, their occultation and emission, and also the random orientation of their angles, coupled with a possible range of accretion rates (Kenyon \& Hartmann 1990) introduces more scatter among the PMS population.

To smooth these effects, the mean of the stellar distribution in Fig. 7c was computed. Interestingly, the mean follows closely the isochrone of 10 Myr shown by a thick line, except at $M_{V}=+1.5$. This is a magnitude point where very often (Rachford \& Canterna 2000; Phelps \& Janes 1993; de Bruijne et al. 2000) a gap appears in many open clusters so that the statistics here may be irrelevant. Therefore we adopt $10 \mathrm{Myr}$ as the mean contraction age of the PMS population in NGC 3293.

\subsubsection{Star formation rate}

Does the fact of finding a 6 Myr age spread among PMS objects indicate the star formation process is not coeval? We examined the point computing individual present-day masses of PMS stars and giving them the corresponding ages interpolating among the Bernasconi \& Maeder (1996) models. Following the Iben \& Talbot (1966) and Adams et al. (1983) definitions and procedures, we estimated the star formation rate (SFR) in the cluster for three different stellar mass bins. The results, shown in Fig. 8, suggest no dependence of the SFR on stellar mass when allowing for the uncertainties involved in that kind of computation (see Stahler 1985). Actually it seems to confirm that the stellar formation in NGC 3293 took place over a very short period of time, mostly between 7 and 10 Myr ago.

\subsubsection{Nuclear and contraction ages}

The mean contraction age of the entire PMS population becomes comparable to the mean nuclear age deduced from stars

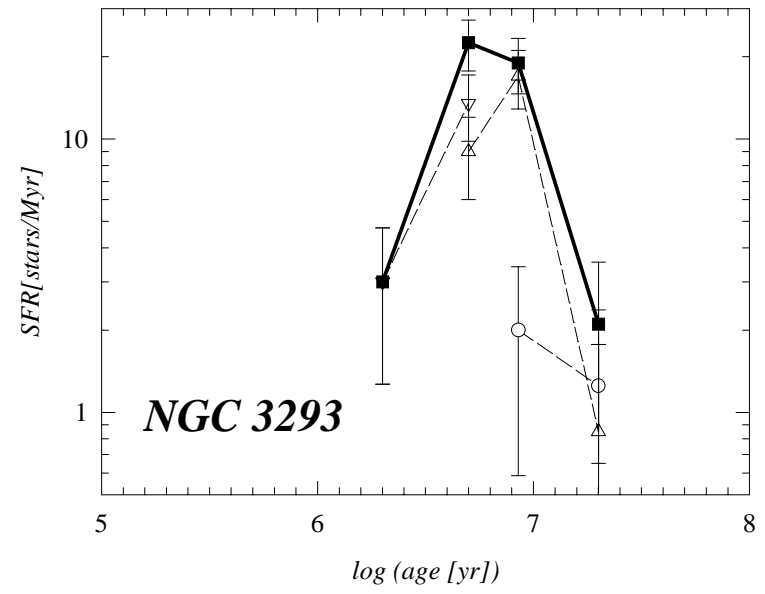

Fig. 8. Star formation rate (SFR) in NGC 3293 from Bernasconi \& Maeder (1996) evolutionary models. Circles, upward triangles and downward triangles indicate SFR values for stars with masses in the intervals $1.0-1.5,1.5-2.0$ and $2.0-2.5 \mathcal{M}_{\odot}$ respectively. Thick line (black squares) represents the total SFR. Error bars are from Poisson statistic.

with $M_{V}<-4$ since there is no substantial difference between the $8 \mathrm{Myr}$ and $10 \mathrm{Myr}$ ages of evolved and PMS stars respectively. Most important is that the early original age discrepancy of 20 Myr found by HM82 is now strongly reduced. On the other hand, as mentioned above, companion stars (Preibisch \& Zinnecker 1999) overestimate the luminosity of a given star (e.g., underestimating the luminosity of an M0 star by a factor of 2 reduces its age by a factor of 4 and a factor of 2 for a G5 star). Also, the net effect of accretion stellar discs, as was mentioned above, introduces scatter in luminosities that produce uncertainties of a factor of 2-3 in the age of an individual object (Kenyon \& Hartmann 1990). We are confident therefore that the $2 \mathrm{Myr}$ difference found between the mean contraction age and the nuclear age is irrelevant in view of the mentioned effects.

Moreover, the $0.2^{\mathrm{m}}$ error in the distance modulus can reduce or increase the difference between both ages without affecting the nuclear age, which remains almost invariable. The "turn on" point position (see Fig. 7c) is not very accurately determined but, allowing for the above uncertainties, it can be placed at $M_{V} \approx+2$ corresponding to a stellar mass $\sim 2 \mathcal{M}_{\odot}$ with a contraction age of $7.9 \mathrm{Myr}$ close to the nuclear age.

\subsection{Cluster LF and IMF}

\subsubsection{The Luminosity Function}

The cluster LF gives the fraction of stars in each absolute magnitude bin of size $\Delta M_{V}=1$. The grey histogram in Fig. 9 shows the LF of NGC 3293 where known cluster binaries (see Table 3) were all corrected by 0.75 and two stars were counted instead of one. For comparison purposes we included also the cluster LF earlier determined by HM82 and the cluster overall LF found by Phelps \& Janes (1993). Except for the strong dip present at $M_{V}=-2$ in the HM82 LF, there are no important differences among the three LFs down to $M_{V} \approx+1$. 


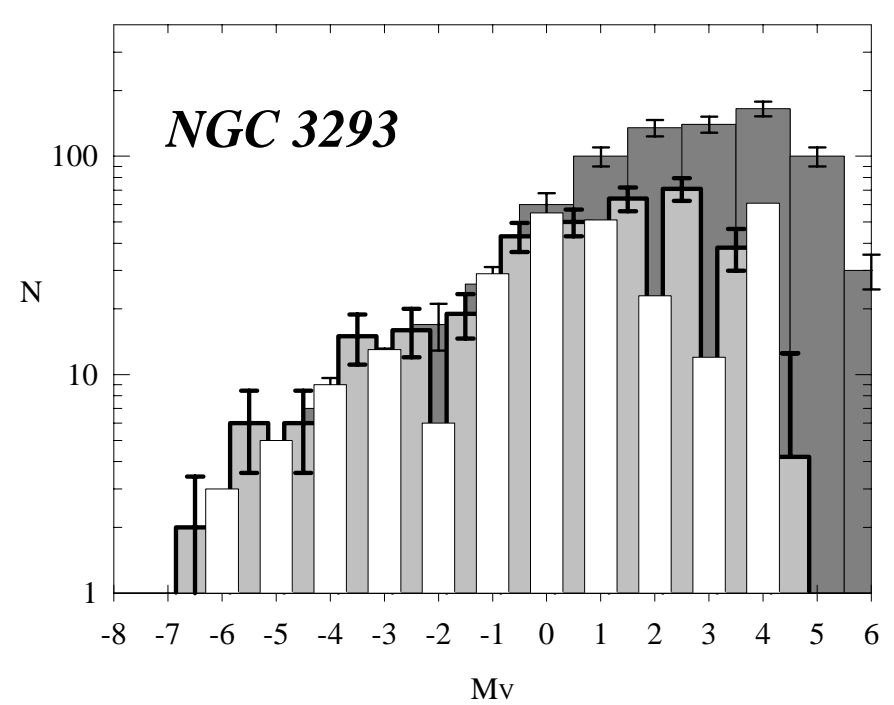

Fig. 9. Luminosity Function of NGC 3293 (grey histogram). Error bars are from Poisson statistic and the subtraction process. The white histogram is the LF from HM82 and the dark-grey one is the combined LF of several young open clusters from Phelps \& Janes (1993).

From $M_{V} \approx+1$, HM82 LF differs from ours, as the sharp dip at $M_{V} \approx+3$ is not revealed by our data. This confirms the suspicion of HM82 that the dip they found is an artifact produced by a combination of incompleteness and eye-estimation of field faint star magnitudes. Interestingly, our LF as well as HM82's show an apparent low number of stars for $M_{V}>0$ when compared to the Phelps \& Janes estimation: ours, in particular, shows a flattening in the range $0<M_{V}<+3-+4$. Notice that all stars below $M_{V} \approx+2$ are PMS objects as we have already stated and their luminosities do not represent the luminosities they will have in the ZAMS; depending on the star location, the present star luminosity and the final in the ZAMS will differ for more than $1^{\mathrm{m}}$, changing the LF shape. Therefore, the cluster LF is only comparable to other obtained from main sequence objects (Wilner \& Lada 1991) in the range $-7<M_{V}<+2$.

\subsubsection{The initial mass function}

Stellar masses were derived using an interpolation process (Baume et al. 1994) converting the $M_{V},(B-V)_{0}$ and $(U-B)_{0}$ values into $\log L$ and $\log T_{\text {eff }}$ first, where mass is assigned in this theoretical plane. The code uses bolometric corrections from Schmidt-Kaler and interpolates among evolutionary tracks reconstructing the path of a given star backwards to its original point in the ZAMS. Bernasconi \& Maeder (1996) tracks were used for masses $<2.5 \mathcal{M}_{\odot}$ and Girardi et al. (2000) tracks for stars above that mass limit. Appropriate mass bins were adopted to distribute stellar masses as shown in Table 4 and the mass points are depicted on top of Fig. 10 with the $\sqrt{N}$ count bars. To get the slope of the cluster IMF $(x)$ we used a weighted least squares method applied to different mass ranges whose results are included at the bottom of Table 4 . The lowest mass bin has been excluded from calculations as it may be affected by effects described below. The fitting of the

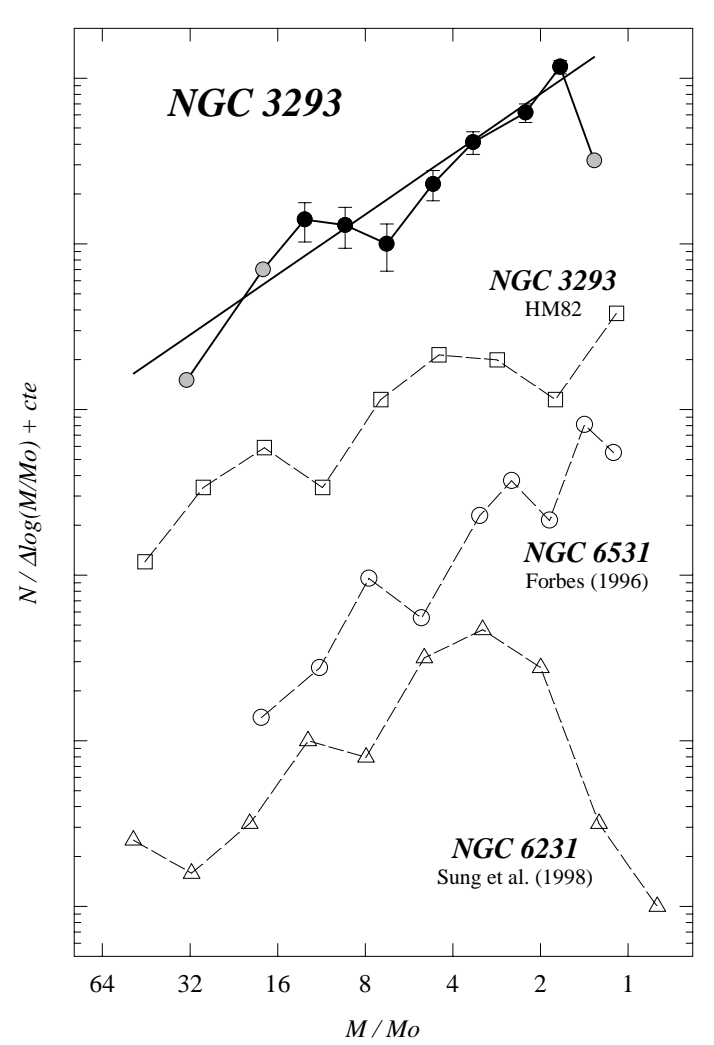

Fig. 10. Initial Mass Function (IMF). The IMF obtained for NGC 3293 is shown at the top (error bars are from Poisson statistics). The weighted least square fitting for the mass range $1.41<\mathcal{M} / \mathcal{M}_{\odot}<$ 16.0 is also presented (points not included in the fit are indicated with grey symbols). Other IMFs for comparable clusters are also shown.

most massive stars gives a steep slope with a high error value $(x=1.6 \pm 0.5)$, however the portion that includes low and intermediate mass stars yields a flatter slope $(x=1.2 \pm 0.2)$. The last value is closer to the typical slope for field stars $(x=1.35)$ and only marginally comparable to the $x=0.9$ found by HM82.

Figure 10 also includes IMFs of clusters of similar ages and the earlier IMF of NGC 3293 determination made by HM82. Interesting common features are shown by the three clusters in the figure. There is always an increasing star number till a dip or flat zone appears. The mass range of the dip is not the same in the three clusters. While ours happens at $8 \mathcal{M}_{\odot}$, it happens at $10 \mathcal{M}_{\odot}$ in the HM82 IMF. Coincident with ours is the dip at $8 \mathcal{M}_{\odot}$ in NGC 6231 but the most strange location corresponds to the IMF of NGC 6531 whose dip happens at $6 \mathcal{M}_{\odot}$. The pattern is not easy to explain but the differences in the mass may reflect differences in the method of assigning masses on the ZAMS according to the evolutionary models used.

\subsubsection{Uncertainties in the LF and the IMF}

There are three main sources of uncertainties in the determination of both the LF and the IMF: the field star correction, the incompleteness of the photometry and the unresolved binaries. Other effects of course do contribute, but the above mentioned are the most difficult to assess. For the removal of the star field contamination, we are confident of the goodness of our method 
Table 4. The initial mass function.

\begin{tabular}{ccc}
\hline \hline$\Delta \mathcal{M} / \mathcal{M}_{\odot}$ & $\Delta M_{V}^{a}$ & $N^{b} / \Delta \log \left(\mathcal{M} / \mathcal{M}_{\odot}\right)$ \\
\hline $22.63-45.25$ & $-7.0--6.0$ & $1.5 \pm 1.2$ \\
$16.00-22.63$ & $-6.0--4.2$ & $7.0 \pm 2.6$ \\
$11.31-16.00$ & $-4.2--3.1$ & $14.0 \pm 3.7$ \\
$8.00-11.31$ & $-3.1--2.2$ & $13.0 \pm 3.6$ \\
$5.66-8.00$ & $-2.2--1.3$ & $10.0 \pm 3.2$ \\
$4.00-5.66$ & $-1.3--0.5$ & $23.0 \pm 4.8$ \\
$2.83-4.00$ & $-0.5-+0.4$ & $41.0 \pm 6.4$ \\
$2.00-2.83$ & $+0.4-+1.6$ & $62.0 \pm 8.0$ \\
$1.41-2.00$ & $+1.6-+3.3$ & $117.0 \pm 10.8$ \\
$1.00-1.41$ & $+3.3-+5.0$ & $31.8 \pm 8.3$ \\
\hline$\Delta \mathcal{M} / \mathcal{M}_{\odot}$ & $x$ & \\
\hline $1.41-45.25$ & $-1.23 \pm 0.13$ & \\
$1.41-16.00$ & $-1.21 \pm 0.18$ & \\
$8.00-45.25$ & $-1.63 \pm 0.52$ & \\
\hline
\end{tabular}

Notes: ${ }^{a}$ Approximated values valid for the evolutionary status of NGC 3293. ${ }^{b}$ Known binaries and incompleteness effects were considered.

and the choice of the areas. Incompleteness is a more complex phenomenon to quantify. An analysis of the completeness factors was presented in Sect. 2 and to remove in part this effect, we applied those factors to the number of stars in each bin of our LF. However, our conclusions may still be affected by incompleteness in the lowest luminosity bin. This uncertainty was minimised in our estimation of the LF that was set in the range from -7 to +2 and was also minimised in the computation of the IMF by excluding the lowest mass bin. Unresolved binaries, however, may influence the entire mass range. We will just describe the probable effect on our determination, as the issue has been treated by many workers and detailed calculations have been already performed. Under the hypothesis that stellar masses of binary components are randomly distributed, Sagar \& Richtler (1991) studied the mass range 2-14 $\mathcal{M}_{\odot}$ finding that, if a fraction of $50 \%$ binaries is present in a given cluster, its IMF slope undergoes a flattening of 0.2 for an initial slope of 1.5. Lower initial slopes are less flattened, according to these authors. Another analysis made by Kroupa et al. $(1991,1992)$ and Kroupa \& Gilmore (1992) also dealt with the point reaching the conclusion that if a Salpeter model is assumed, results lead to an apparent deficiency of low mass stars. This effect is probably the one producing the apparent deficiency of stars in the LF of NGC 3293 when compared to the overall LF of Phelps \& Janes (as marginally shown in Fig. 9).

\section{The search for stars with $\mathrm{H} \alpha$ emission}

One of the aims of this work is the detection of stars with $\mathrm{H}_{\alpha}$ emission. To assess whether a star shows $\mathrm{H}_{\alpha}$ emission, we used the $\mathrm{H}_{\alpha}$ (on) $-\mathrm{H}_{\alpha}$ (off) (hereafter $\Delta \mathrm{H}_{\alpha}$ ) index. Similar indices has been already used in other works (Adams et al. 1983; Sung et al. 1998). We want to mention that due to poor weather conditions, the seeing during the exposures of $\mathrm{H}_{\alpha}$ frames was large $\left(2^{\prime \prime}-2^{\prime \prime} .5\right)$ producing distortions in the final photometry of the stars, especially those with close companions. That, probably, yielded the high errors generated by DAOPHOT, especially in the $\mathrm{H}_{\alpha}$ (off) frames.
Figure $11 \mathrm{~b}$ shows the histogram (white) of $\Delta \mathrm{H}_{\alpha}$ values including all stars. The dark-grey one contains only stars with errors $<0.10$ and was fitted with a Gaussian distribution function over the interval $-0.5<\Delta \mathrm{H}_{\alpha}<0.9$ with mean value $\left\langle\Delta \mathrm{H}_{\alpha}\right\rangle=0.21$ and standard deviation $\sigma_{\Delta \mathrm{H}_{\alpha}}=0.18$. The mean value, which is assumed to correspond to non-emission objects, is close to the values found by Adams et al. (1983) in NGC 2264 (0.30) and NGC 7089 (0.23). However, the dispersion among our data is over twice the value 0.08 found in NGC 2264 by them. Following the reasoning of Adams et al., we identify a probable $\mathrm{H}_{\alpha}$ emission star when its index value is $\Delta \mathrm{H}_{\alpha}<-0.34$ (further than $3 \sigma$ from the mean value) and a likely emission star if it is $\Delta \mathrm{H}_{\alpha}<-0.50$ (further than $4 \sigma)$. All stars showing evidence of emission were plotted with white symbols in the photometric diagrams. It is interesting to mention the clear separation of stars with $\mathrm{H}_{\alpha}$ emission when $\Delta \mathrm{H}_{\alpha}$ values are plotted against the $V-R$ index (see Fig. 11a). We are aware that this procedure only detect stars with high $\mathrm{H}_{\alpha}$ emission while most of PMS stars show weak $\mathrm{H}_{\alpha}$ emission, making their detection much harder (Preibisch \& Zinnecker 1999).

Nineteen stars, listed in Table 5, show evidence of $\mathrm{H}_{\alpha}$ emission. Their spatial location in the NGC 3293 area (shown in Fig. 1) is interesting as eleven of them lie on the external border of the cluster and just another three are inside the cluster limits. From the spatial point of view there is no concentration of emission stars towards the cluster centre, which suggests that the surrounding material of PMS stars in the cluster centre, that usually should produce $\mathrm{H}_{\alpha}$ emission, has already been swept away by the powerful radiation field of the most massive stars. Such a possibility has been suggested to explain the lack of emission stars in NGC 6231 (Sung et al. 1998) and in the Upper Scorpius OB Association (Preibisch \& Zinnecker 1999). If this is true, the rest of the PMS stars included in the cluster area should be weak-lined PMS stars only detectable as X-ray emitters (Montmerle 1996).

\section{Discussion}

Uncertainties in the memberships and also in the stellar age assignment make difficult the interpretation of the star formation process in an open cluster. These uncertainties can lead to a wrong estimate of the star formation rate and therefore to a misinterpretation of the entire process. We attempted to reduce such uncertainties by verifying the reliability of the field star sample used to remove the contribution of field stars and proving that no mass segregation is present in NGC 3293. Moreover, when removing the field contribution from the CMDs of NGC 3293 we chose limits which define the PMS band in terms of both extension and width at a high level of credibility so that only a few stars might have been lost by this procedure. So, we do not expect that our conclusions are seriously affected (qualitatively speaking).

As noticed above, the lack of coincidence between the mean stellar distribution of PMS stars and a given isochrone has been historically used as evidence against the coeval hypothesis (Iben \& Talbot 1966); in the present case there is a strong coincidence. There is a $2 \mathrm{Myr}$ difference between the 


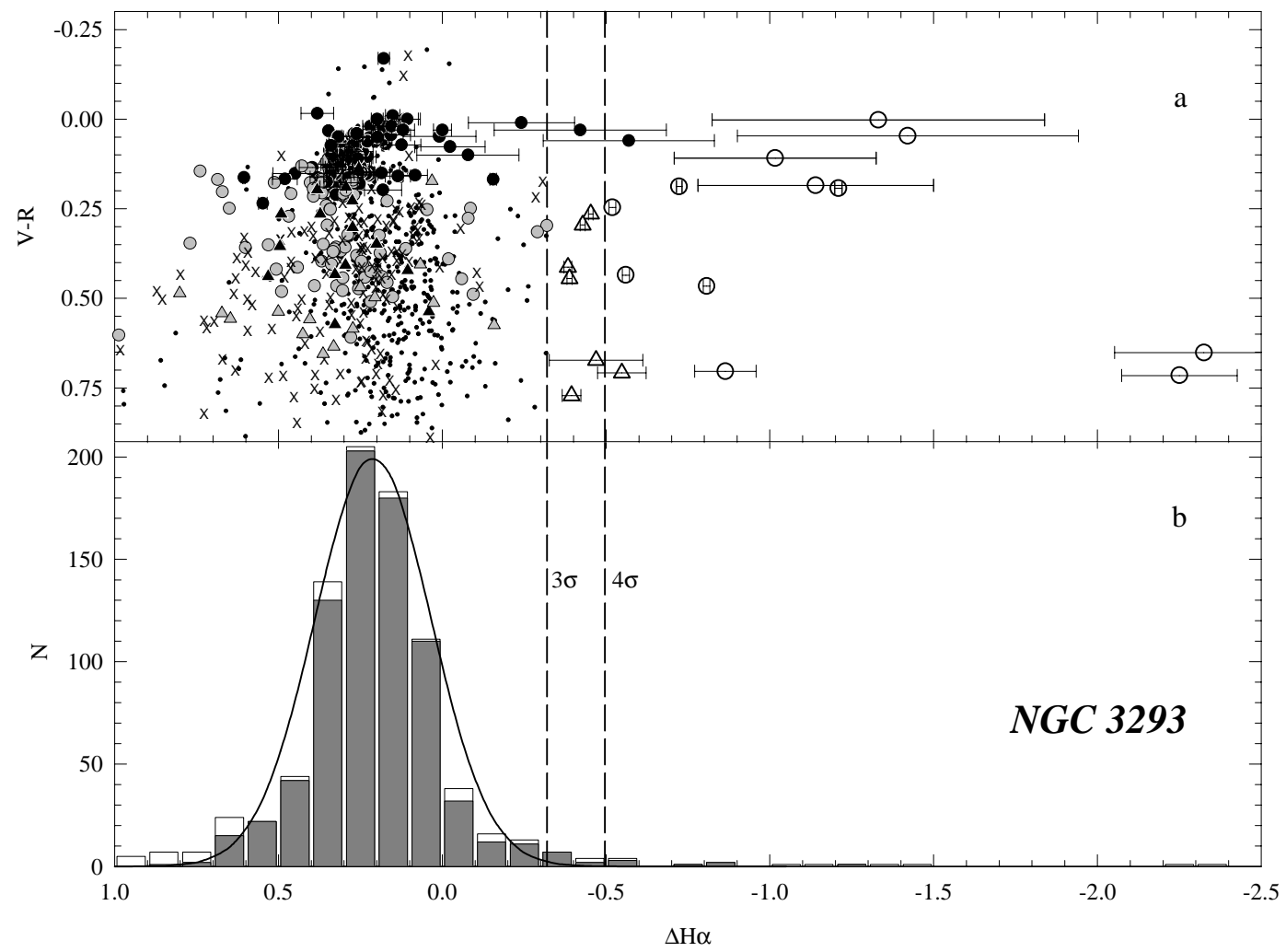

Fig. 11. a) $V-R$ vs. $\Delta \mathrm{H}_{\alpha}$ diagram. Symbols as in Fig. 2a. Error bars from DAOPHOT task for "lm1" and $\mathrm{H}_{\alpha}$ emission stars are also indicated. b) $\Delta \mathrm{H}_{\alpha}$ distribution. White histogram corresponds to all stars with $\Delta \mathrm{H}_{\alpha}$ index and the dark-grey one only includes those with index errors $<0.1$. Solid curve is a Gaussian distribution function fitted to dashed histogram (see Sect. 5). Dashed lines are $3 \sigma$ and $4 \sigma$ limits for $\mathrm{H}_{\alpha}$ emission stars selection.

Table 5. Likely and probable $\mathrm{H}_{\alpha}$ emission stars.

\begin{tabular}{|c|c|c|c|c|c|c|c|c|c|c|}
\hline \multicolumn{11}{|c|}{ Likely $\mathrm{H}_{\alpha}$ emission stars. } \\
\hline \# & $\#_{\mathrm{T}}$ & $\#_{\text {HM }}$ & $x$ & Y & V & $U-B$ & $B-V$ & $V-R$ & $V-I$ & $\Delta \mathrm{H}_{\alpha}$ \\
\hline 31 & 28 & - & 199.7 & 340.9 & 10.27 & -0.66 & 0.01 & 0.00 & 0.05 & $-1.33::$ \\
\hline 63 & - & 277 & 196.9 & 85.5 & 12.02 & -0.28 & 0.22 & 0.18 & 0.39 & $-1.14::$ \\
\hline 67 & 117 & - & 124.6 & 570.0 & 12.10 & $-0.38:$ & 0.28 & 0.05 & 0.44 & $-1.42::$ \\
\hline 85 & - & 264 & 208.8 & 170.1 & 12.34 & -0.48 & 0.10 & 0.11 & 0.23 & $-1.02::$ \\
\hline 297 & - & 287 & 506.0 & 130.0 & 14.56 & 0.20 & 0.31 & 0.19 & 0.43 & -0.72 \\
\hline 324 & - & 286 & 546.9 & 125.0 & 14.69 & 0.25 & 0.31 & 0.19 & 0.42 & -1.21 \\
\hline 361 & - & - & 236.4 & 702.4 & 14.93 & & & 0.25 & & -0.52 \\
\hline 397 & - & 288 & 487.3 & 131.0 & 15.22 & 0.40 & 0.77 & 0.47 & 0.87 & -0.81 \\
\hline 438 & - & 275 & 312.3 & 64.0 & 15.45 & 0.22 & 0.70 & 0.43 & 0.86 & -0.56 \\
\hline 819 & - & - & 816.0 & 195.5 & 17.15 & . & . & 0.72 & . & $-2.25::$ \\
\hline 989 & - & - & 602.4 & 765.1 & 17.72 & . & . & 0.65 & . & $-2.33::$ \\
\hline 1544 & - & - & 771.5 & 519.2 & 19.62 & . & . & 0.70 & . & $-0.86:$ \\
\hline \multicolumn{11}{|c|}{ Probable $\mathrm{H}_{\alpha}$ emission stars. } \\
\hline \# & $\#_{\mathrm{T}}$ & $\#_{\mathrm{HM}}$ & $x$ & Y & V & $U-B$ & $B-V$ & $V-R$ & $V-I$ & $\Delta \mathrm{H}_{\alpha}$ \\
\hline 271 & - & - & 285.0 & 722.4 & 14.40 & . & . & 0.26 & . & -0.45 \\
\hline 391 & - & - & 351.9 & 723.8 & 15.16 & . & . & 0.44 & . & -0.39 \\
\hline 458 & - & - & 147.0 & 665.3 & 15.58 & $0.13:$ & $0.72:$ & 0.30 & $0.80:$ & -0.43 \\
\hline 648 & - & - & 170.2 & 663.8 & 16.47 & 0.46 : & 0.87 : & 0.41 & 0.97 : & -0.38 \\
\hline 926 & - & - & 323.4 & 91.2 & 17.52 & $0.95::$ & 1.28 & 0.77 & 1.50 & -0.39 \\
\hline 1448 & - & - & 667.3 & 722.6 & 19.20 & . & 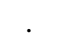 & 0.71 & 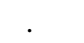 & $-0.55:$ \\
\hline 1599 & - & - & 634.7 & 748.2 & 19.93 & . & . & 0.67 & 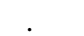 & -0.47:: \\
\hline
\end{tabular}

Notes: \# $_{\mathrm{T}}$ and $\#_{\mathrm{HM}}$ means star numbers from TGHH80 and HM82 respectively.

-Colon (:) and double colon (::) indicate data with errors larger than 0.04 and 0.10 respectively. 
mean nuclear age and the mean contraction age, but it can be explained in terms of the various effects already mentioned. There is no chance either that the age difference between massive and PMS stars reaches the high value of $20 \mathrm{Myr}$ indicated by HM82. That stars in NGC 3293 were all formed in a short period of time is additionally supported by the "turn-on" mass location at $\sim 2 \mathcal{M}_{\odot}$ (compatible with a contraction time ranging from 6 to $12 \mathrm{Myr}$ ) and by the most evolved star in the cluster, the red super-giant, which is 10-12 Myr old, thus equaling the mean age of PMS stars.

The computation of the SFR confirms the above suggestions in the sense that no total increasing SFR, as proposed in other clusters (e.g. NGC 6530 and NGC 2264, Iben \& Talbot 1966) is evident in NGC 3293. Indeed, the picture of this cluster could be similar to the re-interpretation of NGC 2264 and 6530 data (from Iben \& Talbot 1966) made by Stahler (1985). This author found no obvious mass-age correlation but that the star formation in these two clusters took place over a period of time from 5 to 12 Myr. A similar result has been reported for the Upper Scorpius OB Association (Preibisch \& Zinnecker 1999; Preibisch et al. 2002) and for the ScorpiusCentaurus OB Association (Mamajek et al. 2002) where no evidence was found for a real age spread among PMS stars, as the star formation proceeded for a short but intense burst of a few millions years, probably triggered by a supernova event.

\section{Conclusions}

We have investigated the open cluster NGC 3293 area with deep broad band and $\mathrm{H}_{\alpha}$ photometry obtaining a picture of its main sequence structure down to $M_{V} \approx+4.5$. The picture demonstrates that the upper part of its sequence is composed of stars evolving off the ZAMS, the mid one mostly includes stars on the ZAMS and the lower main sequence consists of stars that are placed above the ZAMS, becoming a PMS population.

Clear indications confirming the existence of a PMS population in this cluster are presented after a very careful removal of the field star contamination and also from the finding of $\mathrm{H}_{\alpha}$ emission stars. Our analysis yielded that NGC 3293 is placed at a distance $d=2750 \mathrm{pc}$ and has a $4^{\prime} .1$ angular radius $(3.3 \mathrm{pc})$. The absorption law affecting the cluster is normal although it is close to the northern part of the HII region NGC 3372. In this last particular place, abnormal extinction laws have been found (e.g. Vázquez et al. 1996; Tapia et al. 2002; Carraro et al. 2002). Following the common pattern of clusters in this galactic region, NGC 3293 shows, however, differential reddening surely produced by intracluster material.

Superposition of modern isochrones indicates this object has a nuclear age of $8 \mathrm{Myr}$ and almost a similar mean value of $10 \mathrm{Myr}$ was found among its faintest PMS stars. Therefore, the star formation in NGC 3293 appears to be coeval. Hints that star formation still continues (although probably at a low rate) in NGC 3293 is given by the detection of stars showing $\mathrm{H}_{\alpha}$ emission. These stars are not concentrated towards the cluster centre but they tend to lie in the cluster periphery determined by star counts. The most likely explanation for this lack of concentration may be found in the fact that the radiation field of massive stars has swept away the material that surrounds the PMS stars.

The computation of the cluster LF did not show a strong dip in its structure nor the halo structure of faint stars, as previously suggested by HM82. As in many other young clusters (Tr 14, Vázquez et al. 1996; NGC 6231, Baume et al. 1999; or Pismis 20, Vázquez et al. 1995), we found that the IMF of NGC 3293 has a slope value $x=1.2 \pm 0.2$. This value is of the same order as the one found by Massey et al. (1995) who investigated many open clusters and associations in the Galaxy, the LMC and the SMC, finding a mean slope values of $1.1 \pm 0.1$.

Acknowledgements. The authors acknowledge the financial support from the Facultad de Ciencias Astronómicas y Geofísicas de La UNLP and the IALP-CONICET. Special thanks are given to Dr. Garrison for the allocation of telescope time at UTSO and to the CASLEO staff for the technical support. We are indebted to our careful referee for his suggestions to improve this work. G.B. gratefully acknowledges the collaboration of the Università di Padova (Italy) during a postdoctoral grant.

This article is partially based in the Digitized Sky Survey that was produced at the Space Telescope Science Institute under US government grant NAG W-2166. Original plate material is copyright the Royal Observatory Edinburgh and the Anglo-Australian Observatory. This research has made use of the SIMBAD database, operated at CDS, Strasbourg, France.

\section{References}

Abt, H. A. 1979, ApJ, 230, 485

Adams, M. T., Strom, K. M., \& Strom, S. E. 1983, ApJS, 53, 893

Balona, L. A., \& Crampton, D. 1974, MNRAS, 166, 203

Balona, L. A. 1975, MmRAS, 78, 51

Balona, L. A. 1994, MNRAS, 267, 1060

Balona, L. A., \& Engelbrecht, C. 1981, Workshop on Pulsating B stars, p. 195, ed. G. V. O. N., \& C. Sterken, Nice Observatory

Balona, L. A., \& Engelbrecht, C. 1983, MNRAS, 202, 293

Baume, G., Paoli, S., Vázquez, R. A., \& Feinstein, A. 1994, RMxAA, 29,212

Baume, G. 1999, Ph.D. Thesis, Universidad Nacional de La Plata

Baume, G., Vázquez, R. A., \& Feinstein, A. 1999, A\&A, 137, 233

Battinelli, P., Capuzzo-Dolcetta, R., \& Nesci, R. 1991, MemSAIt, 62, 915

Bernasconi, P. A., \& Maeder, A. 1996, A\&A, 307, 829

Bessell, M. S., \& Stringfellow, G. S. 1993, ARA\&A, 31, 433

Carraro, G. 2002, MNRAS, 331, 785

Carraro, G., Ventura, P., Romanielo, M., \& Patat, F. 2002, A\&A, submitted

Conti, P. S., \& Underhill, A. B. 1988, in O stars, \& Wolf-Rayet stars, Monograph Series of non-thermal phenomena in stellar atmospheres, CNRS and NASA, ed. P. S. Conti, \& A. B. Underhill

Cousins, A. W. J. 1978, MNSSA, 37, 62

Chen, B., Carraro, G., Torra, J., \& Jordi, C. 1998, A\&A, 331, 916

de Bruijne, J. H. J., Hoogerwerf, R., \& de Zeeuw, P. T. 2000, ApJ, 544, L65

Dean, J. F., Warren, P. R., \& Cousins, A. W. J. 1978, MNRAS, 183, 569

Deeg, H. J., \& Ninkov, Z. 1996, A\&AS, 119, 221

ESA 1997, The Hipparcos and Tycho Catalogues, ESA SP-1200

Feast, M. W. 1958, MNRAS, 118, 618

Feinstein, A., \& Marraco, H. G. 1980, PASP, 92, 266 
Flaccomio, E., Micela, G., Sciortino, S., Favata, F., Corbally, C., \& Tomaney, A. 1999, A\&A, 345, 521

Forbes, D. 1996, AJ, 112, 1073

Girardi, L., Bressan, A., Bertelli, G., \& Chiosi, C. 2000, A\&AS, 141, 371

Hartigan, P., Strom, K. M., \& Strom, S. E. 1994, ApJ, 427, 961

Herbst, W., \& Miller, D. P. 1982, AJ, 87, 1478

Hillenbrand, L. A., Massey, P., Strom, S. E., \& Merrill, K. M. 1993, AJ, 106, 1906

Iben, I., \& Talbot, R. J. 1966, ApJ, 144, 968

Janes, K. A., Tilley, C., \& Lingå, G. 1988, AJ, 95, 771

Kenyon, S. J., \& Hartmann, L. W. 1990, ApJ, 349, 197

Kroupa, P., Tout, C. A., \& Gilmore, G. 1991, MNRAS, 251, 293

Kroupa, P., Gilmore, G., \& Tout, C. A. 1992, AJ, 103, 1602

Kroupa, P., \& Tout, C. A. 1992, MNRAS, 259, 223

Lada, C. J., Margulis, M., \& Deaborn, D. 1984, ApJ, 285, 141

Lada, E. A., Strom, K. M., \& Meyers, P. C. 1993, in Prototstars and Planets III, ed. E. H. Levy, \& J. I. Lunine (Tucson: University of Arizona Press), 245

Lada, E. A., \& Lada, C. J. 1995, AJ, 109, 1682

Landolt, A. U. 1992, AJ, 104, 340

Mamajek, E. E., Meyer, M. R., \& Liebert, J. 2002, AJ, 124, 1670

Massey, P., Johnson, K. E., \& DeGioia-Eastwood, K. 1995, ApJ, 454, 151

Meynet, G., Mermilliod, J.-C., \& Maeder, A. 1993, A\&AS, 98, 477

Mermilliod, J.-C. 1976, A\&A, 53, 289

Montmerle, T. 1996, in Ninth Cambridge Workshop on Cool Stars, Stellar Systems, and the Sun, ed. R. Pallavicini, \& A. K. Dupree, ASP Conf. Ser. 109 (San Francisco: ASP), 405

Patat, F., \& Carraro, G. 2001, MNRAS, 325, 1591

Phelps, R. L., \& Janes, K. A. 1993, AJ, 106, 1870

Preibisch, T., \& Zinnecker, H. 1999, AJ, 117, 2381

Preibisch, T., Brown, A. G. A., Bridges, T., Guenther, E., \& Zinnecker, H. 2002, AJ, 124, 404
Prisinzano, L., Carraro, G., Piotto, G., Seleznev, A. F., Stetson, P. B., \& Saviane, I. 2001, A\&A, 369, 851

Rachford, B. J., \& Canterna, R. 2000, AJ, 119, 1296

Sagar, R., \& Richtler, T. 1991, A\&A, 250, 324

Sanner, J., \& Geffert, M. 2001, A\&A, 370, 87

Scalo, J. 1986, Fund. Cos. Phys., 11, 1

Schild, R. E. 1970, ApJ, 161, 855

Schmidt-Kaler, Th. 1982, in Landolt-Bornstein VI/2b

Shobbrook, R. R. 1980, MNRAS, 192, 821

Shobbrook, R. R. 1983, MNRAS, 205, 1215

Stahler, S. W. 1985, ApJ, 293, 207

Stahler, S. W., \& Fletcher, A. B. 1991, MemSAIt, 62, 767

Stetson, P. B. 1987, PASP, 99, 191

Sung, H., Bessell, M. S., \& Lee, S.-W. 1998, AJ, 115, 734

Tapia, M., Roth, M., Vázquez, R. A., \& Feinstein, A. 2002, MNRAS, submitted

Turner, D. G., Grieve, G. R., Herbst, W., \& Harris, W. E. 1980, AJ, 85, 1193

van den Bergh, S., \& Sher, D. 1960, Pub. of the DDO, 2, 203

Vázquez, R. A., \& Feinstein, A. 1991, A\&AS, 90, 317

Vázquez, R. A., Baume, G., Feinstein, A., \& Prado, P. 1994, A\&AS, 106,339

Vázquez, R. A., Will, J.-M., Prado, P., \& Feinstein, A. 1995, A\&AS, 111,85

Vázquez, R. A., Baume, G., Feinstein, A., \& Prado, P. 1996, A\&AS, 116,75

Vázquez, R. A., Baume, G., Feinstein, A., \& Prado, P. 1997, A\&AS, 124,13

Vázquez, R. A., \& Baume, G. 2001, A\&A, 371, 908

Walker, M. F. 1957, ApJ, 125, 636

Walker, M. F. 1961, ApJ, 133, 438

Wilner, D. J., \& Lada, C. J. 1991, AJ, 102, 1050 